\title{
Grassmannian connection between three- and four-qubit observables, Mermin's contextuality and black holes
}

\author{
Péter Lévay, ${ }^{a}$ Michel Planat ${ }^{b}$ and Metod Saniga ${ }^{c}$ \\ ${ }^{a}$ Department of Theoretical Physics, Institute of Physics, \\ Budapest University of Technology and Economics, \\ H-1521 Budapest, Hungary \\ ${ }^{b}$ Institut FEMTO-ST, CNRS, \\ 32 Avenue de l'Observatorie, F-25044 Besançon, France \\ ${ }^{c}$ Astronomical Institute, Slovak Academy of Sciences, \\ SK-05960 Tatranská Lomnica, Slovak Republic \\ E-mail: levay@phy.bme.hu, michel.planat@femto-st.fr, msaniga@astro.sk
}

ABSTRACT: We invoke some ideas from finite geometry to map bijectively 135 heptads of mutually commuting three-qubit observables into 135 symmetric four-qubit ones. After labeling the elements of the former set in terms of a seven-dimensional Clifford algebra, we present the bijective map and most pronounced actions of the associated symplectic group on both sets in explicit forms. This formalism is then employed to shed novel light on recently-discovered structural and cardinality properties of an aggregate of three-qubit Mermin's "magic" pentagrams. Moreover, some intriguing connections with the so-called black-hole-qubit correspondence are also pointed out.

Keywords: Discrete and Finite Symmetries, Black Holes in String Theory, Differential and Algebraic Geometry

ARXiv EPRINT: 1305.5689 


\section{Contents}

1 Introduction 1

2 The geometry of the three-qubit Pauli group 4

3 Clifford labeling of the context space $\quad 7$

4 Planes, trivectors and the Grassmannian $\operatorname{Gr}(6,3) \quad 12$

5 Mapping the context space to symmetric four-qubit operators 16

$\begin{array}{llr}6 & \text { Mermin's pentagrams } & 20\end{array}$

7 A link with the Black-Hole-Qubit correspondence $\quad 27$

8 Conclusions $\quad 31$

\section{Introduction}

Generalized Pauli groups are widely used in the study of multipartite quantum systems associated with finite-dimensional Hilbert spaces. For $N$-partite systems these groups are built from $N$-fold tensor products of the familiar $2 \times 2$ Pauli spin matrices and the $2 \times 2$ identity matrix. Their principal applications lie within a rapidly evolving field of quantum information, where they are mainly related to quantum error correction codes [1]. Such codes are constructed within the framework of the so-called stabilizer formalism [1-4], making use of a simple fact that two elements (observables) in a Pauli group are either commuting, or anticommuting. This physically important property is then encoded into the mathematical structure of a $2 \mathrm{~N}$-dimensional vector space over the two-element field, endowed naturally with a symplectic structure.

Within the past few years, many important ramifications of this idea have appeared. In particular, it has been realized that the commutation algebra for $N$-qubit systems is encapsulated in a hierarchical structure of totally isotropic subspaces of this symplectic vector space, namely a symplectic polar space of rank $N$ and order two $-\mathcal{W}(2 N-1,2)$ [5-7]. Because the sets of pairwise commuting operators are represented by such subspaces, this observation lends naturally itself to a finite geometric analysis of (various aggregates of) Mermin squares and Mermin's pentagrams [8-10], objects which furnish very economical proofs of Bell-Kochen-Specker-like theorems on hidden variables [11, 12].

Another interesting application of this idea concerns the recently-discovered BlackHole-Qubit Correspondence (BHQC; for a recent comprehensive review, see [13]). It has been observed that the structure of certain black hole entropy formulas, coming from charged extremal black hole solutions of effective supergravities stemming from toroidal 
compactifications of string theory, can elegantly be described by distinguished finite pointline incidence structures $[14,15]$. Truncations of these incidence structures to substructures called geometric hyperplanes (which in special cases form configurations like Mermin squares) have been found to correspond to truncations of the relevant supergravities featuring black hole solutions with a restricted set of charges. It turns out that the set of points of these incidence geometries can be mapped to the set of charges the particular supergravity model supports, and their sets of lines to the monomials in the entropy formula presented as a polynomial invariant [15]. Physically, the charges have their origin in wrapping configurations of extended objects (membranes) on special submanifolds of the extra-dimensions [16]. It has also been demonstrated [15] that for a physically consistent realization of charges in a finite-geometric context, these incidence structures should be labeled in a noncommutative manner by elements of the generalized Pauli group for threequbits. Moreover, since different string theories are connected by a web of dualities [16], it may happen that a particular labeling within a framework suggested by one particular string theory should be replaced by another labeling, suggested by its dual counterpart. Hence, such dualities in string theories strongly hint at alternative realizations of the same finite-geometric structures. Thus, on the one hand, one can map the point-set of an incidence geometry to the set of observables and its line-set to tuples of pairwise commuting observables. On the other hand, one can equally well adopt a dual view, regarding a single point as a set of mutually commuting observables, and a line as a tuple of such sets whose pairwise overlapping obeys a prescribed set of rules. A pattern very similar to this has already appeared [17] in an investigation of the structure of the $E_{7(7)}$-symmetric black hole entropy formula of $N=8$ supergravity, based on the incidence structure of the Fano plane.

Surprisingly, this idea has also emerged in investigations of the Bell and Bell-KochenSpecker theorems on hidden variables. There, it is usually emphasized that different sets of mutually commuting observables can be interpreted as representatives of physical situations associated with different possible experimental arrangements/set-ups. If in a hypothetical hidden-variables-theory the result of an observation depends not only on the state of the system, but also on the complete disposition of the apparatus, the corresponding theory is called contextual. It has already been established (see, for example, [8-10] and references therein) that our finite geometries also provide a promising formal footing for dealing with contextuality and associated "context spaces".

A major theme of this paper is the study of the structure of such context space for threequbits. Our reason for deciding to conduct a detailed investigation of this special case is as follows. First, for three-qubits the invariance group of the symplectic form which governs the commutation structures of the corresponding operators is $\operatorname{Sp}(6,2)$. As observed by one of us [18], and elaborated in more detail by [19], this group is related to the Weyl group of the exceptional group $E_{7}, W\left(E_{7}\right)$, as $W\left(E_{7}\right) / \mathbb{Z}_{2}=\operatorname{Sp}(6,2) . W\left(E_{7}\right)$ is the physically important subgroup representing electric-magnetic duality inside the full duality group for toroidal compactifications of string- and $M$-theories to four dimensions [20]. Hence, a clear understanding of representations of this group on objects like our context space can foster a deeper understanding of the BHQC. Second, the elements of context space for three-qubits are heptads of pairwise commuting operators, whose total number amounts 
to 135. From these heptads one can extract three- and four-tuples of observables that represent, respectively, basic building blocks of Mermin squares and Mermin pentagrams. In order to have a deeper, computer-free understanding of recent observations made on such objects $[8-10,15]$, as a first step it is vital to present a broader finite-geometric setting for these structures.

Since the main aim of the present paper is to set such a finite geometric ground for further applications in connection with the BHQC and more general issues of quantum contextuality, we shall also give an explicit form of the bijection between the three-qubit context space, viz. the above-mentioned set of 135 heptads of pairwise commuting observables, and the set of 135 symmetric four-qubit operators. In a finite geometric language, this is a bijection between the 135 maximal totally isotropic subspaces of the polar space $\mathcal{W}(5,2)$ and the 135 points of the hyperbolic quadric $\mathcal{Q}^{+}(7,2)$ fully embedded in the polar space $\mathcal{W}(7,2)$. Though this mapping is well known in the mathematics literature [21-23], mainly as the spin embedding or the spin module for the group $\operatorname{Sp}(6,2)$, its explicit form - to the best of our knowledge - has neither been worked out in detail, nor employed in (mathematical) physics as yet. From the physical point of view, this bijection may in the future play a role very similar to that of the famous Klein correspondence (see, e. g., [24]), which over the complex numbers has already been used in twistor quantization methods of space-time geometry $[25,26]$.

The paper is organized as follows. Section 2 sets the stage for our investigations by summarizing the basics of the finite-geometric background needed later on. Although this summary will be presented merely for the three-qubit case, it generalizes trivially to $\mathrm{N}$ qubits. In section 3 , in the spirit of $[27,28]$, we label the 135 heptads (whose set will be denoted by $\mathcal{I}$ and referred to as the context space in the sequel) by the elements of a seven-dimensional Clifford algebra. Here, the action of the associated symplectic group $\operatorname{Sp}(6,2)$ on this context space is also discussed. In section 4 , the context space is related to the space of separable trivectors satisfying Plücker relations and an additional constraint demanding compatibility with the symplectic polarity. The trivectors arising in this way and encapsulating information on $\mathcal{I}$ are the primitive trivectors. In section 5 , we endow the binary 14-dimensional vector space of primitive trivectors with a symplectic form and show that this space can naturally be expressed as a direct sum of an 8- and a 6-dimensional vector space over $\mathbb{Z}_{2}$. We will further demonstrate that the 8-dimensional space can be identified with the space of real four-qubit observables. Since the latter space comprises exactly 135 symmetric guys, a bijection will be established between these and the 135 heptads of the context space. Employing this bijection, we will subsequently calculate the irreducible [21-23] action of $\operatorname{Sp}(6,2)$ on the space of four-qubit observables. In section 6, we shall first apply our formalism to get deeper insights into the nature of the space of Mermin's pentagrams and to furnish an elegant, computer-fee explanation of some recent findings in this respect $[8,10]$. In section 7 , as a second interesting application of our formalism, by reiterating an observation of [19] we shall show that our space of contexts can be related to the set of possible embeddings of seven copies of the three-qubit SLOCC group [29] inside the exceptional group $E_{7}$ - an idea that has originally been introduced within the context of the BHQC $[17,30]$. Finally, section 8 is reserved for concluding remarks. 


\section{The geometry of the three-qubit Pauli group}

Let us consider the set of three-qubit observables acting on the state space for three-qubits, $\mathcal{H} \equiv \mathbb{C}^{2} \otimes \mathbb{C}^{2} \otimes \mathbb{C}^{2}$. Such observables are of the form $A_{1} \otimes A_{2} \otimes A_{3}$, where the operators $A_{1}, A_{2}, A_{3}$ are elements from the set $\left\{ \pm I, \pm \sigma_{x}, \pm \sigma_{y}, \pm \sigma_{y}\right\} \equiv\{ \pm I, \pm X, \mp i Y, \pm Z\}$. Here, $\sigma_{x}, \sigma_{y}, \sigma_{z}$ are the standard $2 \times 2$ Pauli spin matrices and $I$ is the $2 \times 2$ identity matrix. In what follows, we shall consider instead the real operators of the form $\mathcal{A}_{1} \otimes \mathcal{A}_{2} \otimes \mathcal{A}_{3}$, where $\mathcal{A}_{1}, \mathcal{A}_{2}, \mathcal{A}_{3}$ are elements from the set $\mathcal{P}_{1} \equiv\{ \pm I, \pm X, \pm Y, \pm Z\} . \mathcal{P}_{1}$ is a group and will be called the Pauli group for a single qubit. Notice that the operators $I, X, Z$ are real and symmetric, and the operator $Y$ is real and antisymmetric. Elements of the form $\mathcal{A}_{1} \otimes \mathcal{A}_{2} \otimes \mathcal{A}_{3}$, where $\mathcal{A}_{1}, \mathcal{A}_{2}, \mathcal{A}_{3} \in \mathcal{P}_{1}$, are elements of $\mathcal{P}_{3}$, the three-qubit Pauli group. (The following considerations can be straightforwardly generalized to $N$ qubits.)

An arbitrary element $x$ of $\mathcal{P}_{3}$ can be written in the form

$$
x=(-1)^{s}\left(Z^{a_{1}} X^{b_{1}} \otimes Z^{a_{2}} X^{b_{2}} \otimes Z^{a_{3}} X^{b_{3}}\right) \equiv(s, v)=\left(s, a_{1}, b_{1}, a_{2}, b_{2}, a_{3}, b_{3}\right) \in \mathcal{P}_{3},
$$

where each superscript can acquire two values, 0 and 1 . The product of two elements $x, x^{\prime} \in \mathcal{P}_{3}$ is

$$
x x^{\prime}=\left(s+s^{\prime}+\sum_{i=1}^{3} a_{i}^{\prime} b_{i}, a_{1}+a_{1}^{\prime}, \ldots, b_{3}+b_{3}^{\prime}\right) .
$$

Hence, two elements of $\mathcal{P}_{3}$ commute if, and only if,

$$
\sum_{i=1}^{3}\left(a_{i} b_{i}^{\prime}+a_{i}^{\prime} b_{i}\right)=0 .
$$

The commutator subgroup of $\mathcal{P}_{3}$ coincides with its center $Z(\mathcal{P})=\{I \otimes I \otimes I,-I \otimes I \otimes I\}$; hence, the central quotient $V_{3}=\mathcal{P}_{3} / Z\left(\mathcal{P}_{3}\right)$ is an Abelian group which - by virtue of $(2.2)$ — is also a six-dimensional vector space over $\mathbb{Z}_{2}$, i.e. $V_{3} \equiv \mathbb{Z}_{2}^{6}$. Moreover, the left-hand-side of (2.3) defines on $V_{3}$ a symplectic form

$$
\langle\cdot, \cdot\rangle: V_{3} \times V_{3} \rightarrow \mathbb{Z}_{2}, \quad\left(v, v^{\prime}\right) \mapsto\left\langle v, v^{\prime}\right\rangle \equiv \sum_{i=1}^{3}\left(a_{i} b_{i}^{\prime}+b_{i} a_{i}^{\prime}\right) .
$$

The elements of the vector space $\left(V_{3},\langle\cdot, \cdot\rangle\right)$ are equivalence classes corresponding to pairs of the form $\left\{\mathcal{A}_{1} \otimes \mathcal{A}_{2} \otimes \mathcal{A}_{3},-\mathcal{A}_{1} \otimes \mathcal{A}_{2} \otimes \mathcal{A}_{3}\right\}$, i.e. they are three-qubit operators defined up to a sign. In the sequel, we shall employ a short-hand notation $\mathcal{A}_{1} \mathcal{A}_{2} \mathcal{A}_{3} \equiv\left\{\mathcal{A}_{1} \otimes\right.$ $\left.\mathcal{A}_{2} \otimes \mathcal{A}_{3},-\mathcal{A}_{1} \otimes \mathcal{A}_{2} \otimes \mathcal{A}_{3}\right\}$. Alternatively, we will also refer to this object as an element $v$ of $\left(V_{3},\langle\cdot, \cdot\rangle\right)$.

Since any single-qubit operator $\mathcal{A}$ can be written, sign disregarded, in the form $\mathcal{A}=$ $Z^{a} X^{b}$, where $a, b \in \mathbb{Z}_{2}$, one can associate with it a two component vector $(a, b) \in V_{1} \equiv \mathbb{Z}_{2}^{2}$. Hence, we have

$$
I \mapsto(00), \quad X \mapsto(01), \quad Y \mapsto(11), \quad Z \mapsto(10) .
$$

For a three-qubit operator we adopt the following ordering convention

$$
\mathcal{A}_{1} \mathcal{A}_{2} \mathcal{A}_{3} \leftrightarrow\left(a_{1}, a_{2}, a_{3}, b_{1}, b_{2}, b_{3}\right) \in V_{3} .
$$


Hence, for example,

$$
\{X \otimes Y \otimes Z,-X \otimes Y \otimes Z\} \leftrightarrow X Y Z \leftrightarrow(0,1,1,1,1,0)
$$

and the canonical basis vectors in $V_{3}$ are associated to three-qubit operators as follows

$$
Z I I \leftrightarrow e_{1}=(1,0,0,0,0,0), \ldots, I I X \leftrightarrow e_{6}=(0,0,0,0,0,1)
$$

With respect to this basis, the matrix of the symplectic form is

$$
J_{\mu \nu} \equiv\left\langle e_{\mu}, e_{\nu}\right\rangle=\left(\begin{array}{llllll}
0 & 0 & 0 & 1 & 0 & 0 \\
0 & 0 & 0 & 0 & 1 & 0 \\
0 & 0 & 0 & 0 & 0 & 1 \\
1 & 0 & 0 & 0 & 0 & 0 \\
0 & 1 & 0 & 0 & 0 & 0 \\
0 & 0 & 1 & 0 & 0 & 0
\end{array}\right), \quad \mu, \nu=1,2, \ldots, 6
$$

Since the dimensionality of $V_{3}$ is even and the symplectic form is non-degenerate, the group preserving this symplectic polarity is isomorphic to the group $\operatorname{Sp}(6,2)$. This group acts on the row vectors of $V_{3}$ via $6 \times 6$ matrices $S \in \operatorname{Sp}(6,2)$ from the right, leaving the matrix $J$ invariant

$$
v \mapsto v S, \quad S J S^{t}=J .
$$

It is known that $|\operatorname{Sp}(6,2)|=1451520=2^{9} \cdot 3^{4} \cdot 5 \cdot 7$ and that this group is generated by transvections [19] $T_{w} \in \operatorname{Sp}(6,2), w \in V_{3}$ of the form

$$
T_{w}: V_{3} \rightarrow V_{3}, \quad v \mapsto T_{w} v=v+\langle v, w\rangle w
$$

which is indeed symplectic,

$$
\left\langle T_{w} u, T_{w} v\right\rangle=\langle u, v\rangle .
$$

There is a surjective homomorphism from $W\left(E_{7}\right)$ to $\operatorname{Sp}(6,2)$ with kernel $\mathbb{Z}_{2}$. This homomorphism provides an interesting link between exceptional groups and three-qubit systems. Within the context of Quantum Information this intriguing link was first noticed by one of us [18], and it is also related to the Black-Hole-Qubit Correspondence [17, 30]. A particularly nice elaboration of this homomorphism can be found in the paper of Cerchiai and van Geemen [19].

Given an element $v \in V_{3}$, let us define the quadratic form

$$
Q_{0} \equiv \sum_{i=1}^{3} a_{i} b_{i} .
$$

It is easy to check that for vectors representing symmetric operators $Q_{0}(v)=0$ and for antisymmetric ones $Q_{0}(v)=1$; moreover,

$$
\langle u, v\rangle=Q_{0}(u+v)+Q_{0}(u)+Q_{0}(v) .
$$


This quadratic form can be regarded as the one labeled by the trivial element of $V_{3}$, corresponding to the trivial observable $I I I$. There are, however, 63 other quadratic forms $Q_{w}$ associated with the symplectic form $\langle\cdot \mid \cdot\rangle$ labeled by 63 nontrivial elements of $V_{3}$

$$
\langle u, v\rangle=Q_{w}(u+v)+Q_{w}(u)+Q_{w}(v)
$$

they are defined as

$$
Q_{w}(v)=Q_{0}(v)+\langle w, v\rangle^{2},
$$

where the square can be omitted as we work over $\mathbb{Z}_{2}$. For more details on these quadratic forms, we refer the reader to $[28,31]$; here, we merely note that a form labeled by a symmetric observable $\left(Q_{0}(w)=0\right)$ represents in the associated projective space $P G(5,2)$ a locus of points satisfying $Q_{w}(v)=0$, which is also called a hyperbolic quadric and usually denoted by $\mathcal{Q}^{+}(5,2)$. On the other hand, the locus of points satisfying $Q_{w}(v)=0$ where $Q_{0}(w)=1$ is denoted by $\mathcal{Q}^{-}(5,2)$ and called an elliptic quadric. These quadrics can trivially be generalized to $N$-qubits. Thus, for example, a hyperbolic quadric in $P G(7,2)$, $\mathcal{Q}^{+}(7,2)$, given by the locus $Q_{0}(v)=0, v \in V_{4}$, is just the quadric whose points correspond to the symmetric four-qubit observables.

By virtue of the special character of the field $\mathbb{Z}_{2}$, a one-dimensional subspace of $V_{3}$ (consisting of elements of the form $\lambda v$ where $\lambda \in \mathbb{Z}_{2}$ and $v \in V_{3}$ ) is spanned by a unique nonzero vector $v \in V_{3}$. Hence, the 63 points of the projective space $P G(5,2)$ can be identified with the 63 nonzero vectors of $V_{3}$. Since the vector space $V_{3}$ underlying $P G(5,2)$ is equipped with a symplectic form, one can determine on any subspace $W$ of $P G(5,2)$ a symplectic polarity $\perp$, i.e. the map

$$
W \mapsto W^{\perp}, \quad \text { where } \quad W^{\perp}=\left\{v \in V_{3} \mid w J v^{T}=0, \forall w \in W\right\},
$$

and call such subspace non-isotropic, isotropic or totally isotropic according as $W \cap W^{\perp}=$ $\{0\}, W \cap W^{\perp} \neq\{0\}$ or $W \subset W^{\perp}$, respectively. A maximal totally isotropic subspace is isomorphic to $P G(2,2)$ - the Fano plane. The space of all totally isotropic subspaces of $(P G(5,2), \perp)$ is the symplectic polar space of rank three and order two, $\mathcal{W}(5,2)$; it contains 63 points, 315 lines and 135 planes (see, e. g., [32]).

Let us illustrate these abstract concepts in terms of the physically relevant structures of three-qubit operators (defined up to a sign). The 63 points of $P G(5,2)$ (as well as of $\mathcal{W}(5,2))$ are all 63 nontrivial operators of $\mathcal{P}_{3} . \quad W$ is any subset of pairwise commuting operators and $W^{\perp}$ is the set of operators commuting with each member of this particular subset. A line $L \in P G(5,2)$ is an object of the form $L=\lambda v+\mu u$, where $u, v \in P G(5,2)$ and $\lambda, \mu \in \mathbb{Z}_{2}$. It contains the following three points $u, v, u+v$ and thus corresponds to a triple of operators such that the product of any two of them yields the third one. A line (as well as a plane) of $P G(5,2)$ belongs to $\mathcal{W}(5,2)$ if, and only if, the corresponding operators are also pairwise commuting. A plane of $\mathcal{W}(5,2)$ represents a heptad, i.e. a maximum set, of mutually commuting three-qubit observables. An illustrative example is furnished by the heptad $\{I I X, I X I, I X X, X I I, X I X, X X I, X X X\}$; the seven lines of this plane have 
the following representatives

$$
\begin{array}{lllr}
\{I I X, I X I, I X X\}, & \{I I X, X I I, X I X\}, & \{I I X, X X I, X X X\},\{I X I, X I I, X X I\}, \\
\{X X X, X I I, I X X\}, & \{X X I, X I X, I X X\}, & \{X X X, X I X, I X I\} .
\end{array}
$$

The central object of our reasoning in the subsequent sections will be the set of 135 heptads of mutually commuting elements of $\mathcal{P}_{3}$, aka the set of 135 planes of $\mathcal{W}(5,2) .{ }^{1}$ Before proceeding further, and given the fact that the language of finite geometry employed in the sequel is not a commonplace in either quantum information or high energy physics, we think the reader finds convenient a short summary of basic cardinality characteristics of key finite geometrical objects involved:

\begin{tabular}{|lrr|}
\hline Name of Object & No. of Points & No. of Maximal Subspaces \\
\hline Projective (Fano) plane PG(2,2) & 7 & 7 \\
Projective space PG $(3,2)$ & 15 & 15 \\
Hyperbolic (Klein) quadric $\mathcal{Q}^{+}(5,2)$ & 35 & $2 \times 15=30$ \\
Symplectic polar space $\mathcal{W}(5,2)$ & 63 & 135 \\
Hyperbolic quadric $\mathcal{Q}^{+}(7,2)$ & 135 & $2 \times 135=270$ \\
Symplectic polar space $\mathcal{W}(7,2)$ & 255 & 2295 \\
Split Cayley hexagon of order two & 63 & 63 \\
\hline
\end{tabular}

\section{Clifford labeling of the context space}

Our next goal is to find a convenient labeling of the elements of $\mathcal{I}$ and then describe the action of $\operatorname{Sp}(6,2)$ on this set. To begin with, we first label the 63 nontrivial three-qubit operators in terms of the generators of a $\operatorname{Cliff}(7)$ algebra

$$
\left\{\Gamma_{a}, \Gamma_{b}\right\}=-2 \delta_{a b}, \quad a, b=1,2,3,4,5,6,7 .
$$

A possible choice of generators satisfying eq. (3.1) is

$$
\begin{array}{llll}
\Gamma_{1}=I \otimes I \otimes Y, & \Gamma_{2}=Z \otimes Y \otimes X, & \Gamma_{3}=Y \otimes I \otimes X, & \Gamma_{4}=Y \otimes Z \otimes Z, \\
\Gamma_{5}=X \otimes Y \otimes X, & \Gamma_{6}=I \otimes Y \otimes Z, & \Gamma_{7}=Y \otimes X \otimes Z . &
\end{array}
$$

Assuming that $1 \leq a<b<c<\cdots<e<f \leq 7$, we will use the shorthand notation $\pm \Gamma_{a} \Gamma_{b} \Gamma_{c} \cdots \Gamma_{e} \Gamma_{f} \equiv a b c \cdots e f$. When needed, for products we will sometimes use a cyclic reordering (e.g., " $267 " \rightarrow$ " 672 "). Notice that antisymmetric operators can be represented by singlets and doublets of Clifford generators, whereas symmetric ones are expressed in terms of triplets; thus, for example, $\Gamma_{1} \Gamma_{2}=Z \otimes Y \otimes Z$ is an antisymmetric operator and $\Gamma_{1} \Gamma_{2} \Gamma_{5}=Y \otimes I \otimes Y$ is a symmetric one.

In a previous paper [14] we have shown that there is an automorphism of order seven which acts on $\mathcal{P}_{3}$ via conjugation. Under this automorphism the 63 nontrivial threequbit operators split into nine different orbits containing seven operators each. One of the

\footnotetext{
${ }^{1}$ By a slight abuse of notation, we shall use the same symbol, $\mathcal{I}$, for both the sets.
} 
orbits consists of the (3.2) basis vectors of the Clifford algebra. In order to see this, let $\alpha \equiv(1,2, \cdots, 7)$ denote the permutation $1 \mapsto 2 \mapsto \cdots \mapsto 7 \mapsto 1$. Then the matrix $\mathcal{D}(\alpha)$ that acts via conjugation

$$
\mathcal{A}_{1} \otimes \mathcal{A}_{2} \otimes \mathcal{A}_{3} \mapsto \mathcal{D}^{-1}(\alpha)\left(\mathcal{A}_{1} \otimes \mathcal{A}_{2} \otimes \mathcal{A}_{3}\right) \mathcal{D}(\alpha)
$$

and shifts cyclically the generators of our Clifford algebra, i.e. $\Gamma_{1} \mapsto \Gamma_{2} \mapsto \cdots \mapsto \Gamma_{7} \mapsto \Gamma_{1}$, is of the form [14]

$$
\mathcal{D}(\alpha) \equiv\left(\begin{array}{cccc}
P & Q & 0 & 0 \\
0 & 0 & Q & P \\
0 & 0 & Q X & P X \\
P X & Q X & 0 & 0
\end{array}\right), \quad P=\left(\begin{array}{ll}
1 & 0 \\
0 & 0
\end{array}\right), \quad Q=\left(\begin{array}{ll}
0 & 0 \\
0 & 1
\end{array}\right) .
$$

An alternative form of this $8 \times 8$ matrix can be given [14] in terms of the two-qubit CNOT operations familiar from Quantum Information,

$$
\mathcal{D}(\alpha)=\left(C_{12} C_{21}\right)\left(C_{12} C_{31}\right) C_{23}\left(C_{12} C_{31}\right),
$$

where

$$
\begin{aligned}
C_{12}=\left(\begin{array}{llll}
I & 0 & 0 & 0 \\
0 & I & 0 & 0 \\
0 & 0 & 0 & I \\
0 & 0 & I & 0
\end{array}\right), & C_{21}=\left(\begin{array}{llll}
I & 0 & 0 & 0 \\
0 & 0 & 0 & I \\
0 & 0 & I & 0 \\
0 & I & 0 & 0
\end{array}\right), \\
C_{23}=\left(\begin{array}{cccc}
I & 0 & 0 & 0 \\
0 & X & 0 & 0 \\
0 & 0 & I & 0 \\
0 & 0 & 0 & X
\end{array}\right), & C_{31}=\left(\begin{array}{cccc}
P & 0 & Q & 0 \\
0 & P & 0 & Q \\
Q & 0 & P & 0 \\
0 & Q & 0 & P
\end{array}\right) .
\end{aligned}
$$

As every three-qubit operator can be expressed in terms of the basis vectors (3.2) of the Clifford algebra, the nine orbits under $\mathcal{D}(\alpha)$ explicitly read

$$
\begin{aligned}
(1,2,3,4,5,6,7) & \leftrightarrow(I I Y, Z Y X, Y I X, Y Z Z, X Y X, I Y Z, Y X Z), \\
(12,23,34,45,56,67,71) & \leftrightarrow(Z Y Z, X Y I, I Z Y, Z X Y, X I Y, Y Z I, Y X X), \\
(13,24,35,46,57,61,72) & \leftrightarrow(Y I Z, X X Y, Z Y I, Y X I, Z Z Y, I Y X, X Z Y), \\
(14,25,36,47,51,62,73) & \leftrightarrow(Y Z X, Y I I, Y Y Y, I Y I, X Y Z, Z I Y, I X Y), \\
(123,234,345,456,567,671,712), & \leftrightarrow(X Y Y, Z X Z, X X Z, Z Z X, Z X X, Y Z Y, X Z I), \\
(125,236,347,451,562,673,714), & \leftrightarrow(Y I Y, X I Z, Y Y X, Z X I, Y Y Z, I Z X, I Y Y), \\
(135,246,357,461,572,613,724), & \leftrightarrow(Z Y Y, X Z X, X Z Z, Y X Y, I X Z, Y Y I, Z I X), \\
(124,235,346,457,561,672,713), & \leftrightarrow(X X I, I I X, I X X, X I X, X I I, X X X, I X I), \\
(126,237,341,452,563,674,715), & \leftrightarrow(Z I I, Z Z Z, I Z I, I Z Z, Z I Z, I I Z, Z Z I) .
\end{aligned}
$$

Moreover, $\mathcal{D}(\alpha)$ belongs to $\mathrm{SO}(8)$ and is orthogonal, $\mathcal{D}^{T}(\alpha)=\mathcal{D}^{-1}(\alpha)$; hence, under its conjugate action symmetric operators are mapped to symmetric, and antisymmetric operators to antisymmetric ones. We have four orbits of antisymmetric operators and five orbits of symmetric ones. 
Let us also give the $6 \times 6$ matrix representation $D(\alpha)$ of the cyclic shift $\alpha=(1234567)$. According to eq. (2.10), $D(\alpha)$ acts from the right on the elements of $v \in V_{3}=\mathbb{Z}_{2}^{6}$ regarded as six-component row vectors with

$$
D(\alpha)=\left(\begin{array}{cc}
K & 0 \\
0 & L
\end{array}\right), \quad K=\left(\begin{array}{lll}
1 & 1 & 1 \\
0 & 1 & 1 \\
1 & 1 & 0
\end{array}\right), \quad L=\left(\begin{array}{lll}
1 & 1 & 1 \\
1 & 1 & 0 \\
0 & 1 & 1
\end{array}\right),
$$

where off-diagonal blocks contain merely zeros. Clearly, $D(\alpha) J D^{T}(\alpha)=J$ and $D(\alpha)^{7}=1$, which implies that $D(\alpha) \in \operatorname{Sp}(6,2)$ and is of order seven. For example, the action of $D(\alpha)$ yields

$$
(001001) \mapsto(110011) \mapsto(100101) \mapsto(111100) \mapsto(010111) \mapsto(011010) \mapsto(101110),
$$

which corresponds to the orbit given by eq. (3.4).

We already know that $\operatorname{Sp}(6,2)$ is generated by symplectic transvections. However, for the reader's convenience, we shall also give its presentation featuring merely two generators $\alpha$ and $\beta$, one of them being our cyclic shift of order seven and the other a particular element of order two. The presentation in question is ${ }^{2}$

$$
\operatorname{Sp}(6,2)=\left\langle\alpha, \beta \mid \alpha^{7}=\beta^{2}=(\beta \alpha)^{9}=\left(\beta \alpha^{2}\right)^{12}=[\beta, \alpha]^{3}=\left[\beta, \alpha^{2}\right]^{2}=1\right\rangle .
$$

The $6 \times 6$ matrix representation $D(\beta)$ of the generator $\beta$, which acts on $V_{3}$ from the right, is given by

$$
D(\beta)=\left(\begin{array}{llllll}
1 & 0 & 0 & 0 & 0 & 0 \\
0 & 1 & 0 & 0 & 0 & 0 \\
1 & 1 & 1 & 0 & 0 & 1 \\
1 & 1 & 0 & 1 & 0 & 1 \\
1 & 1 & 0 & 0 & 1 & 1 \\
0 & 0 & 0 & 0 & 0 & 1
\end{array}\right) .
$$

This matrix is again symplectic, $D(\beta) J D^{T}(\beta)=J$, and of order two. The action of $D(\beta)$ induced on three-qubit operators, defined up to a sign, has the following form

$$
\begin{aligned}
& I I Z \leftrightarrow Z Z Y, \quad I I Y \leftrightarrow Z Z Z, \quad I X I \leftrightarrow Z Y X, \quad I X X \leftrightarrow Z Y I, \\
& I Z Z \leftrightarrow Z I Y, \quad I Z Y \leftrightarrow Z I Z, \quad I Y I \leftrightarrow Z X X, \quad I Y X \leftrightarrow Z X I, \\
& X I I \leftrightarrow Y Z X, \quad X I X \leftrightarrow Y Z I, \quad X X Z \leftrightarrow Y Y Y, \quad X X Y \leftrightarrow Y Y Z, \\
& X Z I \leftrightarrow Y I X, \quad X Z X \leftrightarrow Y I I, \quad X Y Z \leftrightarrow Y X Y, \quad Y X Z \leftrightarrow X Y Y,
\end{aligned}
$$

with the remaining elements being left invariant. One observes that the transformations above can be obtained via (up to a sign) multiplication by the operator $Z Z X$, which anticommutes with all of the operators appearing in the list; the remaining 31 invariant operators are precisely those that commute with $Z Z X$. Hence, according to eq. (2.11), $D(\beta)$ is just a matrix representative of the transvection defined by $v=(110001) \leftrightarrow Z Z X$.

\footnotetext{
${ }^{2}$ The explicit form of this representation for the generator $\beta$ and a presentation for $G_{2}(2)$ was determined by P. Vrana in his MSc thesis.
} 
Notice also that, unlike $\alpha$, the generator $\beta$ cannot be lifted to a conjugate action on $\mathcal{P}_{3}$ of the (3.3) type via an orthogonal matrix. This is immediately obvious from the fact that $D(\beta)$ maps symmetric operators to antisymmetric ones, and vice versa. We also mention that in terms of the labels referring to our $\operatorname{Cliff}(7)$ algebra, the action of $\beta$ can be summarized as

$$
\begin{aligned}
& (123,7),(237,1),(137,2),(127,3), \\
& (14,156),(15,146),(16,145), \quad(24,256),(25,246),(26,245), \\
& (34,356),(35,346),(36,345), \quad(47,567),(57,467),(67,457) .
\end{aligned}
$$

The above-described $\operatorname{Cliff}(7)$-labeling of three-qubit operators, although being of importance of its own, also leads to a neat description of the context space, $\mathcal{I}$, with two classes of elements of cardinality 105 and 30.

A Fano plane of the first class is of the type $\{7,12,34,56,127,347,567\}$, i.e. it comprises four antisymmetric and three symmetric operators. The pattern clearly shows that the corresponding seven operators are pairwise commuting. In order to also understand the structure of its lines, one notes that $1234567 \leftrightarrow I I I$, and $\Gamma_{a}^{2} \leftrightarrow I I I$. Hence, the double occurrence of any number, as well as the occurrence of all numbers from 1 to 7 , yields the identity. The lines of the Fano plane are thus the combinations $(7,12,127),(7,34,347),(7,56,567)$, and the ones $(127,347,567),(12,34,567),(12,56,347),(34,56,127)$. Notice that the three antisymmetric operators 12, 34, 56 already determine the Fano plane; indeed, they cannot be collinear since $123456 \leftrightarrow 7$. As a consequence, all the planes featuring the operator $7 \leftrightarrow Y X Z$ can be characterized by all the disjoint triples of doublets featuring all the numbers from 1 to 6 . There are 15 doublets, of which 15 such triples can be formed. These 15 triples form the lines of a $P G(3,2)$. Hence, there are 15 planes featuring the operator $Y X Z$ related to this specific $P G(3,2)$ :

$$
\begin{aligned}
& \{7,16,25,34,167,257,347\} \leftrightarrow\{Y X Z, I Y X, Y I I, I Z Y, Y Z Y, I X Z, Y Y X\}, \\
& \{7,16,24,35,167,247,357\} \leftrightarrow\{Y X Z, I Y X, X X Y, Z Y I, Y Z Y, Z I X, X Z Z\}, \\
& \{7,12,34,56,127,347,567\} \leftrightarrow\{Y X Z, Z Y Z, I Z Y, X I Y, X Z I, Y Y X, Z X X\}, \\
& \{7,14,25,36,147,257,367\} \leftrightarrow\{Y X Z, Y Z X, Y I I, Y Y Y, I Y Y, I X Z, I Z X\}, \\
& \{7,16,23,45,167,237,457\} \leftrightarrow\{Y X Z, I Y X, X Y I, Z X Y, Y Z Y, Z Z Z, X I X\}, \\
& \{7,13,25,46,137,257,467\} \leftrightarrow\{Y X Z, Y I Z, Y I I, Y X I, I X I, I X Z, I I Z\}, \\
& \{7,15,26,34,157,267,347\} \leftrightarrow\{Y X Z, X Y Z, Z I Y, I Z Y, Z Z I, X X X, Y Y X\}, \\
& \{7,14,26,35,147,267,357\} \leftrightarrow\{Y X Z, Y Z X, Z I Y, Z Y I, I Y Y, X X X, X Z Z\}, \\
& \{7,12,45,36,127,457,367\} \leftrightarrow\{Y X Z, Z Y Z, Z X Y, Y Y Y, X Z I, X I X, I Z X\}, \\
& \{7,13,24,56,137,247,567\} \leftrightarrow\{Y X Z, Y I Z, X X Y, X I Y, I X I, Z I X, Z X X\}, \\
& \{7,15,24,36,157,247,367\} \leftrightarrow\{Y X Z, X Y Z, X X Y, Y Y Y, Z Z I, Z I X, I Z X\}, \\
& \{7,14,23,56,147,237,567\} \leftrightarrow\{Y X Z, Y Z X, X Y I, X I Y, I Y Y, Z Z Z, Z X X\}, \\
& \{7,12,35,46,127,357,467\} \leftrightarrow\{Y X Z, Z Y Z, Z Y I, Y X I, X Z I, X Z Z, I I Z\}, \\
& \{7,15,23,46,157,237,467\} \leftrightarrow\{Y X Z, X Y Z, X Y I, Y X I, Z Z I, Z Z Z, I I Z\}, \\
& \{7,13,26,45,137,267,457\} \leftrightarrow\{Y X Z, Y I Z, Z I Y, Z X Y, I X I, X X X, X I X\} .
\end{aligned}
$$


Using the cyclic shift and the corresponding action of $\mathcal{D}(\alpha)$, we can generate seven more planes for each member of this set of 15 planes. Hence the number of Fano planes of this kind is $7 \times 15=105$.

A Fano plane of the second class consists solely of symmetric operators, i.e. triples of numbers from 1 to 7 . Since the automorphism group of the Fano plane has order 168, the number of such Steiner triples is $7 ! / 168=30$. The set of triples $124,235,346,457,561,672$ and 713 corresponds to the pairwise commuting set of eq. (3.11), featuring only $X$ and $I$,

$$
\{124,235,346,457,561,672,713\} \leftrightarrow\{X X I, I I X, I X X, X I X, X I I, X X X, I X I\} .
$$

Similarly, the set of triples $126,237,341,452,563,674$ and 715 corresponds to the pairwise commuting set of eq. (3.12), featuring only $Z$ and $I$,

$$
\{126,237,341,452,563,674,715\} \leftrightarrow\{Z I I, Z Z Z, I Z I, I Z Z, Z I Z, I I Z, Z Z I\} .
$$

Because these two particular planes are left invariant by $\mathcal{D}(\alpha)$, no new planes can be generated from them. However, the remaining 28 planes of this class indeed arise from four distinguished ones under the cyclic shift of $\mathcal{D}(\alpha)$. These distinguished planes are

$$
\begin{aligned}
& \{123,147,156,246,257,345,367\} \leftrightarrow\{X Y Y, I Y Y, X I I, X Z X, I X Z, X X Z, I Z X\}, \\
& \{127,135,146,236,245,347,567\} \leftrightarrow\{X Z I, Z Y Y, Y X Y, X I Z, I Z Z, Y Y X, Z X X\}, \\
& \{126,134,157,235,247,367,456\} \leftrightarrow\{Z I I, I Z I, Z Z I, I I X, Z I X, I Z X, Z Z X\}, \\
& \{124,137,156,236,257,345,467\} \leftrightarrow\{X X I, I X I, X I I, X I Z, I X Z, X X Z, I I Z\} .
\end{aligned}
$$

We have mentioned in the preceding section that the symmetric elements of $\mathcal{P}_{3}$ all lie on a particular hyperbolic quadric $\mathcal{Q}^{+}(5,2) \equiv \mathcal{Q}$ of the ambient projective space $P G(5,2)$. We have also found that each plane of the second class features only symmetric elements; hence, all of them must by fully located on this particular quadric. Next, it is well known that the planes lying on any $\mathcal{Q}^{+}(5,2)$ split into two distinct systems, of cardinality 15 each. Employing the famous Klein correspondence between the lines of $P G(3,2)$ and the points of the (Klein quadric) $\mathcal{Q}^{+}(5,2)$ [24], a plane from one system corresponds to the set of lines through a point of $P G(3,2)$, whereas a plane of the other system answers to the set of lines in a plane of $P G(3,2)$. From this correspondence it readily follows that two distinct planes belonging the same system have just a single point in common, whilst two planes of different systems are either disjoint, or share a line. Thus, our two special planes, eq. (3.40) and eq. (3.39), being disjoint, come from different systems. Further, the planes defined by eqs. (3.40), (3.42) and (3.44) are all from the same system, since their pairwise intersection is a single point. Clearly, this property is also exhibited by the remaining 12 planes arising via a repeated action of the automorphism of order seven, which thus complete one system. Similarly, the 15 planes defined by eqs. (3.39), (3.41) and (3.43), together with their 12 cyclically shifted cousins, belong all to the other system. For the reader's convenience, all the 30 planes lying on our Klein quadric $\mathcal{Q}$ will be explicitly listed later on (see section 6).

We conclude this section by noting that the action of $\operatorname{Sp}(6,2)$ on $\mathcal{I}$ is transitive [21], that is, given any two planes from $\mathcal{I}$, one can find an element of $\operatorname{Sp}(6,2)$ that sends one plane to the other. 


\section{Planes, trivectors and the Grassmannian $\operatorname{Gr}(6,3)$}

Having at our disposal a rather detailed description of the context space $\mathcal{I}$ and $\operatorname{Sp}(6,2)$ action on it, we can now proceed to our second major task, namely the issue of mapping bijectively this space into the one of symmetric operators on four qubits. As already mentioned in the introduction, although the explicit form of this mapping has not yet been worked out, the geometric construction underlying it - the so called spin module of the group $\operatorname{Sp}(6,2)$ - is well known in mathematical literature [21-23]. In order to understand this construction, we will first provide another important representation of our context space. Obviously, the set of planes $\mathcal{I}$ can be regarded as a special subset of a total of 1395 planes living in $P G(5,2)$. So, as a first step first, we will characterize this full set of planes in terms of 20 Plücker coordinates, which are related to the independent components of separable trivectors.

The set of planes in $P G(5,2)$ comes from the projectivization of the set of threedimensional subspaces in our six-dimensional vector space $V_{3}=\mathbb{Z}_{2}^{6}$, i.e. from the Grassmannian $\operatorname{Gr}(6,3)$; the projectivization of this latter space will be denoted by $\mathcal{G} r(5,2)$. Each element of $\mathcal{G} r(5,2)$ can be viewed as the left row space of a $3 \times 6$ matrix $(A \mid B)$ of rank 3 , where $A$ and $B$ are $3 \times 3$ matrices, whose entries are taken from $\mathbb{Z}_{2}$. The meaning of this term is as follows. The three rows of the $3 \times 6$ matrix $(A \mid B)$ can be regarded as the three linearly independent vectors spanning a three-dimensional subspace in $V_{3}$, i.e. an element of $\operatorname{Gr}(6,3)$; equivalently, the corresponding points span a plane of $P G(5,2)$, i.e. an element of $\mathcal{G} r(5,2)$. For example, the plane $\{7,12,34,56,127,347,567\}$ of eq. (3.26) is spanned by the observables $12,34,56$, i.e. $Z Y Z, I Z Y, X I Y$. Their corresponding vectors in $V_{3}$ are (111010), (011001), (001101); hence, we have

$$
\{7,12,34,56,127,347,567\} \leftrightarrow(A \mid B), \quad A=\left(\begin{array}{lll}
1 & 1 & 1 \\
0 & 1 & 1 \\
0 & 0 & 1
\end{array}\right), \quad B=\left(\begin{array}{lll}
0 & 1 & 0 \\
0 & 0 & 1 \\
1 & 0 & 1
\end{array}\right)
$$

Under the left action via an element $T \in G L\left(3, \mathbb{Z}_{2}\right)$, we obtain a new $3 \times 6$ matrix $\left(A^{\prime} \mid B^{\prime}\right)=$ $T(A \mid B)$ that, obviously, represents the same plane of $\mathcal{G} r(5,2)$. We express this property symbolically as $(A \mid B) \simeq\left(A^{\prime} \mid B^{\prime}\right)$. As $G L\left(3, \mathbb{Z}_{2}\right)$ is isomorphic to the automorphism group of the Fano plane, it merely permutes the basis elements of a given plane from $\mathcal{G} r(5,2)$. For planes represented by a matrix $(A \mid B)$ such that $B \in G L\left(3, \mathbb{Z}_{2}\right)$, i.e. when $B$ is invertible, one can use the matrix $\left(M \mid I_{3}\right)$ as a representative spanning the same plane. Here, $M=$ $B^{-1} A$ and $I_{3}$ is the $3 \times 3$ identity matrix. For example, the plane defined by eq. (4.1) is characterized by $\operatorname{Det} B=1$; hence, $B \in G L\left(3, \mathbb{Z}_{2}\right)$ and a short calculation yields

$$
(A \mid B) \simeq\left(M, I_{3}\right), \quad M=B^{-1} A=\left(\begin{array}{lll}
0 & 1 & 0 \\
1 & 1 & 1 \\
0 & 1 & 1
\end{array}\right),
$$

and the three rows of the new matrix $\left(M \mid I_{3}\right)$ thus define another triple of operators, namely $X Z I, Z Y Z, I Z Y$. Since, according to eq. (3.26), these operators show up in the list of seven operators and they are not collinear, they span the same plane. On the other hand, all 
planes with coordinates $(A \mid B)$, where $B \notin G L\left(3, \mathbb{Z}_{2}\right)$, will be called planes at infinity. The plane represented by the matrix $\left(I_{3} \mid 0\right)$ will be called the distinguished plane of $P G(5,2)$. This is the plane defined by eq. (3.40), forming an orbit of its own under $\mathcal{D}(\alpha)$. One can show that a plane is at infinity precisely when it has nonzero intersection with this distinguished plane.

In the next step, we shall embed $\mathcal{G} r(5,2)$ into the space of trivectors $\bigwedge^{3} \mathbb{Z}_{2}^{6}$ using the well-known Plücker embedding. An arbitrary trivector can be expressed as

$$
P=\sum_{1 \leq \mu<\nu<\rho \leq 6} P_{\mu \nu \rho} e_{\mu} \wedge e_{\nu} \wedge e_{\rho}
$$

Here, $P_{\mu \nu \rho}$ are $\left(\begin{array}{l}6 \\ 3\end{array}\right)=20$ linearly independent expansion coefficients. Since these 20 numbers are in $\mathbb{Z}_{2}$, the alternating property now means symmetrization as well as vanishing of the diagonal elements. Hence, the $20 P_{\mu \nu \rho}$ s can be extended to a rank three tensor whose indices are symmetric under permutations, but which vanishes when any two indices happen to be the same. An element $P \in \Lambda^{3} \mathbb{Z}_{2}^{6}$ is called separable if it can be written in the form $P=u \wedge v \wedge w$ for some linearly independent elements $u, v, w \in V_{3}$. Hence, a threespace of $\operatorname{Gr}(6,3)$ corresponds to a separable three-form in $\bigwedge^{3} \mathbb{Z}_{2}^{6}$. Equivalently, a plane in $\mathcal{G} r(5,2)$ corresponds to a point in the subset of separable trivectors in the projectivization of $\bigwedge^{3} \mathbb{Z}_{2}^{6}$, which is a 19-dimensional projective space over $\mathbb{Z}_{2}, P G(19,2)$. Explicitly, the Plücker embedding $\theta$ is given by the map

$$
\theta: G r(6,3) \hookrightarrow \bigwedge^{3} \mathbb{Z}_{2}^{6}, \quad(M \mid N)=\left(\begin{array}{cccccc}
u_{1} & u_{2} & u_{3} & u_{4} & u_{5} & u_{6} \\
v_{1} & v_{2} & v_{3} & v_{4} & v_{5} & v_{6} \\
w_{1} & w_{2} & w_{3} & w_{4} & w_{5} & w_{6}
\end{array}\right) \hookrightarrow u \wedge v \wedge w
$$

Using the canonical basis vectors $e_{\mu}$ defined by eq. (2.8), we have

$$
u \wedge v \wedge w=P_{123} e_{1} \wedge e_{2} \wedge e_{3}+P_{124} e_{1} \wedge e_{2} \wedge e_{4}+\cdots+P_{456} e_{4} \wedge e_{5} \wedge e_{6} .
$$

Hence, for a separable trivector, $P_{\mu \nu \rho}$ are the $3 \times 3$ minors of the matrix $(M \mid N)$ obtained by keeping merely the columns of this $3 \times 6$ matrix labelled by the fixed numbers $\mu, \nu, \rho$; they are called the Plücker coordinates of the given plane. Clearly, the Plücker coordinates are not independent. They are subject to quadratic relations, called the Plücker relations. It is known (see, for example, [34]) that an arbitrary $P \in \bigwedge^{3} \mathbb{Z}_{2}^{6}$ is separable if, and only if, its coefficients $P_{\mu \nu \rho}$ satisfy the Plücker relations. In our special case these relations can elegantly be described as follows.

For an arbitrary $3 \times 3$ matrix $M$, let us denote by $M^{\sharp}$ the transposed cofactor matrix of $M$. Then, we have

$$
M M^{\sharp}=M^{\sharp} M=\operatorname{Det}(M) I_{3}, \quad\left(M^{\sharp}\right)^{\sharp}=\operatorname{Det}(M) M, \quad(M N)^{\sharp}=N^{\sharp} M^{\sharp},
$$

and

$$
\operatorname{Det}(M+N)=\operatorname{Det} M+\operatorname{Tr}\left(M N^{\sharp}\right)+\operatorname{Tr}\left(M N^{\sharp}\right)+\operatorname{Det} N \text {. }
$$


For a plane that can be represented in the form $\left(M \mid I_{3}\right)$, the Plücker coordinates can be conveniently arranged as two numbers and two $3 \times 3$ matrices as follows

$$
P_{123} \equiv \operatorname{Det} M, \quad\left(\begin{array}{lll}
P_{156} & P_{256} & P_{356} \\
P_{146} & P_{246} & P_{346} \\
P_{145} & P_{245} & P_{345}
\end{array}\right)=M, \quad\left(\begin{array}{lll}
P_{234} & P_{235} & P_{236} \\
P_{134} & P_{135} & P_{136} \\
P_{124} & P_{125} & P_{126}
\end{array}\right)=M^{\sharp}, \quad P_{456}=1 .
$$

Let us refer to these quantities as the four-tuple

$$
(m, M, N, n) \equiv\left(\operatorname{Det} M, M, M^{\sharp}, 1\right) .
$$

Now, in light of the identities given by (4.6), we have

$$
m M=N^{\sharp}, \quad n N=M^{\sharp}, \quad m n I_{3}=M N .
$$

It can be shown that these expressions, quadratic in Plücker coordinates, are nothing but the usual Plücker relations. We have thus shown that the $\theta$-images of planes of the form $\left(M \mid I_{3}\right)$ obey the Plücker relations. Conversely, it can be shown [33] that if an arbitrary trivector $P$, whose Plücker coordinates are arranged into a four-tuple $(m, M, N, n)$, where

$$
m=P_{123}, \quad M=\left(\begin{array}{lll}
P_{156} & P_{256} & P_{356} \\
P_{146} & P_{246} & P_{346} \\
P_{145} & P_{245} & P_{345}
\end{array}\right), \quad N=\left(\begin{array}{lll}
P_{234} & P_{235} & P_{236} \\
P_{134} & P_{135} & P_{136} \\
P_{124} & P_{125} & P_{126}
\end{array}\right), \quad n=P_{456},
$$

meets the constraints given by (4.10), then $P$ is separable. Hence, eq. (4.10) can be used as a sufficient and necessary condition for the separability of a trivector. Such trivectors can be identified with the Grassmannian $G r(6,3)$ via the Plücker embedding $\theta$. From the projective viewpoint, the set of planes in $P G(5,2)$ is identified with the set of points of a certain algebraic variety of $P G(19,2)$; this variety is defined by eq. (4.10).

Further, one can define, in a complete analogy to what we did in the case of $P G(5,2)$, a symplectic polarity also on $P G(19,2)$. This polarity originates from a symplectic form $\mathcal{B}$ defined on the associated 20-dimensional vector space $\mathcal{V}_{10}$ over $\mathbb{Z}_{2}$,

$$
\mathcal{B}: \mathcal{V}_{10} \times \mathcal{V}_{10} \rightarrow \mathbb{Z}_{2}, \quad\left((m, M, N, n),\left(m^{\prime}, M^{\prime}, N^{\prime}, n^{\prime}\right)\right) \mapsto m n^{\prime}+n m^{\prime}+\operatorname{Tr}\left(M N^{\prime}+N M^{\prime}\right)
$$

Here, the coordinates of a vector of $\mathcal{V}_{10}$ are given in the form of (4.11). Moreover, in analogy to eq. (2.13) we can also define a quadratic form associated with $\mathcal{B}$,

$$
q_{0}: \mathcal{V}_{10} \rightarrow \mathbb{Z}_{2}, \quad(m, M, N, n) \mapsto m n+\operatorname{Tr}(M N)
$$

Notice that, formally, one can regard the vector space $\mathcal{V}_{10}$ as the set of 10 -qubit Pauli operators (defined up to a sign). Then, as usual, the symplectic form $\mathcal{B}$ vanishes for commuting and differs from zero for anticommuting pairs of such operators. Likewise, the quadratic form $q_{0}$ again gives zero for symmetric and one for antisymmetric operators. When $(m, M, N, n)$ represents a separable trivector, one can employ eq. (4.10) to see that there exists a plane represented as $(A \mid B)$ such that

$$
m=\operatorname{Det} A, \quad M=B^{\sharp} A, \quad N=A^{\sharp} B, \quad n=\operatorname{Det} B .
$$


In this special case

$$
q_{0}((m, M, N, n))=\operatorname{Det} A \operatorname{Det} B+\operatorname{Tr}\left(B^{\sharp} A A^{\sharp} B\right)=\operatorname{Det} A \operatorname{Det} B+\operatorname{Det} A \operatorname{Det} B \operatorname{Tr} I_{3}=0 .
$$

This implies that the planes of $P G(5,2)$ are mapped to those points of $P G(19,2)$ that are lying on a certain hyperbolic quadric, viz. the one that accommodates all symmetric operators of 10-qubit Pauli group.

It is obvious that $\mathcal{I}$, being a subset of planes of $P G(5,2)$, will be mapped by $\theta$ to a subvariety of the variety defined by eq. (4.10). In order to find this subvariety, we make use of the symplectic polarity $\perp$ on $P G(5,2)$. Since the Plücker map sends planes to trivectors, planes of our context space will be represented by special trivectors. To see this, we first notice that the action of $\operatorname{Sp}(6,2)$ is no longer irreducible on the 20-dimensional space of trivectors. It can be shown that the 20-dimensional representation of the group, induced by its particular representation on $V_{3}$, decomposes as $20=6 \oplus 14$. In order to properly grasp this decomposition, let us introduce a bivector associated to the symplectic form $J$ in the following way

$$
J=\sum_{1 \leq \mu<\nu \leq 6} J_{\mu \nu} e_{\mu} \wedge e_{\nu}
$$

where $J_{\mu \nu}$ is given by eq. (2.9). Now, $\wedge^{3} V_{3}$ decomposes as

$$
\wedge^{3} V_{3}=J \wedge V_{3}+\wedge_{0}^{3} V_{3}, \quad P_{0} \in \wedge_{0}^{3} V_{3} \quad \text { iff } \quad J \wedge P_{0}=0 .
$$

The trivectors $P_{0} \in \wedge_{0}^{3} V_{3}$ are called primitive and span the 14-dimensional irreducible subspace. Writing out the constraint $J \wedge P_{0}=0$ explicitly shows that, in terms of the components $P_{\mu \nu \rho}$, the condition for primitivity can be expressed as an extra condition on the matrices $M$ and $N$ of eq. (4.11), namely

$$
M^{T}=M, \quad N^{T}=N
$$

that is, these matrices become symmetric. A brute-force calculation shows that each plane from $\mathcal{I}$ is mapped by $\theta$ to a primitive trivector satisfying eq. (4.18). Here is a quick demonstration for the special case when either $\operatorname{Det} A$, or $\operatorname{Det} B$, is nonzero. One first notes that for $(A \mid B) \in \mathcal{I}$ the row vectors are pairwise orthogonal; this implies that $(A \mid B) J(A \mid B)^{T}=0$ and so $A B^{T}=B A^{T}$. If $\operatorname{Det} B \neq 0$, we can use an equivalent description of this plane as $\left(M \mid I_{3}\right)$, where $M=B^{\sharp} A$. Now, $\left(M \mid I_{3}\right) \in \mathcal{I}$, so $M^{T}=M$ and because $N=M^{\sharp}$ (see eq. (4.8)), we have $N^{T}=N$, too. For $\operatorname{Det} A \neq 0$ we use $\left(I_{3} \mid N\right)$ with $N=A^{\sharp} B$ to arrive at the same result.

The upshot of these considerations is as follows. Take a particular plane of $\mathcal{G}(5,2)$, represented in the form $(A \mid B)$. Calculate its Plücker coordinates, and arrange them into a four-tuple $(m, M, N, n)$ using eq. (4.11). If the plane belongs to $\mathcal{I}$, then the corresponding matrices $M$ and $N$ will be symmetric. As a consequence, only 14 Plücker coordinates suffice to represent a plane from $\mathcal{I}$. This implies that the $\theta$-image of $\mathcal{I}$ spans in $P G(19,2)$ a projective subspace of dimension $13, P G(13,2)$. 


\section{Mapping the context space to symmetric four-qubit operators}

Because the vector space associated with the projective subspace representing $\mathcal{I}$ is 14dimensional, we will refer to it as $\mathcal{V}_{7}$. It is instructive to calculate the restriction of the symplectic form $\mathcal{B}$ (eq. (4.12)) to $\mathcal{V}_{7}$. Let $\xi, \xi^{\prime} \in \mathcal{V}_{7}$, where $\xi \equiv(m, M, N, n), \xi^{\prime}=$ $\left(m^{\prime}, M^{\prime}, N^{\prime}, n^{\prime}\right)$ and both $M$ and $N$ are symmetric. By virtue of eq. (4.11), we get

$$
\begin{aligned}
\mathcal{B}\left(\xi, \xi^{\prime}\right)= & P_{123} P_{456}^{\prime}+P_{456} P_{123}^{\prime} \\
& +P_{156} P_{234}^{\prime}+P_{234} P_{156}^{\prime}+P_{246} P_{135}^{\prime}+P_{135} P_{246}^{\prime}+P_{345} P_{126}^{\prime}+P_{126} P_{345}^{\prime} ;
\end{aligned}
$$

that is, because we are over $\mathbb{Z}_{2}$ and all off-diagonal elements of $M$ and $N$ occur in doubles, only the diagonal elements of these matrices are nonzero. This implies that whether two elements of $\mathcal{V}_{7}$ are orthogonal or not is determined merely by eight numbers comprising $m, n$ and the six diagonal elements of $M$ and $N$. Rephrased in the language of Pauli operators, the fact whether two seven-qubit operators commute or not is determined solely by the relevant four-qubit part.

In order to isolate this important four-qubit part, let us split our $\mathcal{V}_{7}$ into an 8- and a 6-dimensional vector subspace as

$$
\mathcal{V}_{7}=\mathcal{V}_{4} \oplus \mathcal{V}_{3}
$$

Here, the elements of $\mathcal{V}_{4}$ are of the form

$$
\left(P_{123}, P_{156}, P_{246}, P_{345}, P_{456}, P_{234}, P_{135}, P_{126}\right)
$$

and the elements of $\mathcal{V}_{3}$ have the following representatives

$$
\left(P_{146}, P_{245}, P_{356}, P_{235}, P_{136}, P_{124}\right)=\left(P_{256}, P_{346}, P_{145}, P_{134}, P_{125}, P_{236}\right) .
$$

Under this ordering of the components for the four-qubit part, the restricted symplectic form $\mathcal{B}$ features a matrix similar to eq. (2.9), where $I_{3}$ is now replaced by the matrix $I_{4}$ in the off-diagonal blocks. Clearly, to an element of $\mathcal{V}_{4}$ one can associate a four-qubit operator defined, up to a sign, as

$$
\left(P_{123}, P_{156}, P_{246}, P_{345}, P_{456}, P_{234}, P_{135}, P_{126}\right)=\left(a_{1} a_{2} a_{3} a_{4} b_{1} b_{2} b_{3} b_{4}\right) .
$$

Here, the pair $\left(a_{i} b_{i}\right), i=1,2,3,4$, corresponds to the $j$-th qubit, with the corresponding operator given by the dictionary furnished by eq. (2.5). It is also important to realize that, according to eq. (4.15), the $\mathcal{V}_{4}$-restriction of the quadratic form (4.13) shows that the relevant four-qubit operators associated to planes from $\mathcal{I}$ are all symmetric. This means that we can establish an explicit mapping from $\mathcal{I}$ to the points of a hyperbolic quadric defined by the zero locus of the quadratic form $q_{0}$ restricted to $P G(7,2)$. Let us denote this hyperbolic quadric by $\mathcal{Q}^{+}(7,2)$. And because we have 135 planes in $\mathcal{I}$ and there are precisely 135 points on the hyperbolic quadric $\mathcal{Q}^{+}(7,2)$ (see, for example, [35]), this mapping should be - and indeed is - a bijection.

In order to establish an explicit form of this bijection, we will proceed as follows. We take our list of planes defined by eqs. (3.24)-(3.44). For each of these 21 representative 
planes, one picks up three non-collinear three-qubit operators. They define three linearly independent row vectors, which we arrange as in eq. (4.4). These vectors then generate the $3 \times 6$ matrix $(A \mid B)$. Subsequently, after calculating minors with columns being just the labels of $P_{\mu \nu \rho}$, we determine the 8 relevant Plücker coordinates of eq. (5.3). Then, using the dictionary given by (5.5), we read off the corresponding four-qubit operator. The results of our calculation can be summarized as

$$
\begin{aligned}
\{7,16,25,34,167,257,347\} & \mapsto Y Y X Z, \\
\{7,16,24,35,167,247,357\} & \mapsto Y I Y X, \\
\{7,12,34,56,127,347,567\} & \mapsto Y I Z Y, \\
\{7,14,25,36,147,257,367\} & \mapsto Y Y I I, \\
\{7,16,23,45,167,237,457\} & \mapsto I Y Z Y, \\
\{7,13,25,46,137,257,467\} & \mapsto I I X Z, \\
\{7,15,26,34,157,267,347\} & \mapsto I Y Y X, \\
\{7,14,26,35,147,267,357\} & \mapsto X X Y Y, \\
\{7,12,45,36,127,457,367\} & \mapsto X X Z X, \\
\{7,13,24,56,137,247,567\} & \mapsto X Z X I, \\
\{7,15,24,36,157,247,367\} & \mapsto Z Z Z X, \\
\{7,14,23,56,147,237,567\} & \mapsto Z Z Y Y, \\
\{7,12,35,46,127,357,467\} & \mapsto Z X I Z, \\
\{7,15,23,46,157,237,467\} & \mapsto Z X X I, \\
\{7,13,26,45,137,267,457\} & \mapsto X Z I Z, \\
\{123,147,156,246,257,345,367\} & \mapsto X X I I, \\
\{127,135,146,236,245,347,567\} & \mapsto Z I Z Z, \\
\{126,134,157,235,247,367,456\} & \mapsto I I I X, \\
\{124,137,156,236,257,345,467\} & \mapsto I I I Z, \\
\{124,235,346,457,561,672,371\} & \mapsto X I I I, \\
\{126,237,341,452,563,674,715\} & \mapsto Z I I I .
\end{aligned}
$$

Note that the last two planes are the special ones, eqs. (3.39)-(3.40), fixed by the automorphism of order seven. The 19 remaining planes generate the remaining elements of $\mathcal{I}$ via this automorphism. We know that this automorphism acts on the left-hand-side of the bijection as a cyclic shift of order seven. In order to find the four-qubit labels of the remaining planes, we need to figure out how this automorphism acts on the right-hand-side.

To this end in view, one observes that the $6 \times 6$ matrix of this automorphism acts on a plane of the form $(A \mid B)$ as

$$
(A \mid B) \mapsto\left(A^{\prime} \mid B^{\prime}\right)=(A K \mid B L),
$$

where $K$ and $L$ are the matrices known from eq. (3.13). Notice that $\operatorname{Det} K=\operatorname{Det} L=1$ and $L^{\sharp}=K^{T}$. Then $m^{\prime}=\operatorname{Det} A^{\prime}=\operatorname{Det} A \operatorname{Det} K=m$, and $n^{\prime}=\operatorname{Det} B^{\prime}=\operatorname{Det} B \operatorname{Det} L=n$. 
Hence, the first and the fourth coordinate in eq. (5.5) does not change,

$$
P_{123}^{\prime}=P_{123}, \quad P_{456}^{\prime}=P_{456} .
$$

Moreover, by virtue of (4.14), we have

$$
M^{\prime}=(B L)^{\sharp} A K=L^{\sharp}\left(B^{\sharp} A\right) K=K^{T} M K, \quad N^{\prime}=(A K)^{\sharp} B L=L^{T} M L .
$$

From the last equations we can extract the following transformation rules for diagonal elements

$$
\begin{aligned}
& P_{156}^{\prime}=P_{156}+P_{345}, \quad P_{246}^{\prime}=P_{246}+P_{156}+P_{345}, \quad P_{345}^{\prime}=P_{156}+P_{246}, \\
& P_{234}^{\prime}=P_{234}+P_{135}, \quad P_{135}^{\prime}=P_{135}+P_{234}+P_{126}, \quad P_{126}^{\prime}=P_{126}+P_{234} .
\end{aligned}
$$

Since we work over $\mathbb{Z}_{2}$, matrices $M$ and $N$ do not exhibit any mixing of diagonal and off-diagonal entries. One can also describe these transformation rules by the $8 \times 8$ matrix

$$
R(\alpha)=\left(\begin{array}{llllllll}
1 & 0 & 0 & 0 & 0 & 0 & 0 & 0 \\
0 & 1 & 1 & 1 & 0 & 0 & 0 & 0 \\
0 & 0 & 1 & 1 & 0 & 0 & 0 & 0 \\
0 & 1 & 1 & 0 & 0 & 0 & 0 & 0 \\
0 & 0 & 0 & 0 & 1 & 0 & 0 & 0 \\
0 & 0 & 0 & 0 & 0 & 1 & 1 & 1 \\
0 & 0 & 0 & 0 & 0 & 1 & 1 & 0 \\
0 & 0 & 0 & 0 & 0 & 0 & 1 & 1
\end{array}\right)
$$

acting from the right on row vectors of (5.5). As this matrix also contains the matrices $K$ and $L$ of eq. (3.13), the transformation rules of the four-qubit symmetric operators under the automorphism of order seven are rather simple. The first (leftmost) qubit is left invariant, whereas the second, third and the fourth operators, regarded altogether as a three-qubit one, are cyclically shifted according to the pattern we already know from eqs. (3.4)-(3.12). Thus, for example, a cyclic shift sends the plane (5.6) to

$$
\{1,27,36,45,127,136,145\} \mapsto Y I I Y
$$

where for the shift of the last three operators of the four-qubit one we took into account eq. (3.4). We have thus completed our task of labeling the elements of the context space, $\mathcal{I}$, in terms of symmetric four-qubit operators. A brief inspection of eqs. (5.6)-(5.26) shows that two planes overlap when the corresponding four-qubit operators are commuting. This is in accordance with the proposition [23] that two points lying on $\mathcal{Q}^{+}(7,2)$ are perpendicular with respect to the symplectic form given by eq. (4.12) if, and only if, the corresponding planes from $\mathcal{I}$ have non-empty intersection. As we have already seen, there are two possibilities for this: either the two planes share a point, or a line. An example of the first case is furnished by the planes (5.7) and (5.8), the second case can be illustrated by the planes (5.6) and (5.7). For an example of two disjoint planes one can consider the 
planes (5.6) and (5.26), for the corresponding four-qubit observables labeling these planes are anticommuting.

At this point we will make a slight digression from our main line of reasoning and consider a spread of planes of $P G(5,2)$, that is, a set of pairwise disjoint planes partitioning its point-set. From the physical point of view, such a spread is a partition of the 63 nontrivial observables of $\mathcal{P}_{3}$ into nine pairwise disjoint heptads. As an illustrative example, we can take the following set

$$
\begin{aligned}
& \{\{X Z Y, Z Y Y, Y X I, Y X Y, Z Y I, X Z I, I I Y\}, \\
& \{Y I I, I Y Y, Y Y Y, I Z X, Y Z X, Y X Z, I X Z\}, \\
& \{Z X X, I X X, Z I I, I Y Z, Z Z Y, I Z Y, Z Y Z\}, \\
& \{Y X Z, I X I, Z I Z, Y I X, X X Y, X I Y, Y X X\}, \\
& \{Z I Y, Z X I, I X Y, Y Y X, X Y Z, Y Z Z, X Z X\}, \\
& \{X X I, Y Y I, Z Z I, X X Z, Y Y Z, I I Z, Z Z Z\}, \\
& \{X Y Y, Z Y X, Y I Z, I Z Z, Z X Y, Y Z I, X X X\}, \\
& \{Z Z X, Z I X, I Z I, X Z Z, Y I Y, X I Z, Y Z Y\}, \\
& \{I Y X, X Y I, I X X, X Y X, X I X, X I I, I Y I\}\}
\end{aligned}
$$

Using our dictionary, we readily find that this spread corresponds to the following set of four-qubit observables

$$
\{Y X Z Y, Y Y I I, I Z X X, I Z X Z, Y Z I Y, I X X I, I X Y Y, Z I Z I, X I Z I\} .
$$

A quick check shows that these observables $\gamma_{r}, r=1,2, \cdots, 9$, are pairwise anticommuting and each squares to $I_{16}$; hence, $\left\{\gamma_{r}, \gamma_{s}\right\}=2 \delta_{r s} I_{16}$, i.e. they form the basis vectors of a Cliff $(9)$. Geometrically speaking, they represent an ovoid of $\mathcal{Q}^{+}(7,2)$ (see, e. g., $[35,36]$ ). It is known that there are 960 such spreads/ovoids, hence the number of possible basis vectors for a Clif $f(9)$ algebra made entirely from symmetric four-qubit observables is 960 as well. We also mention that the notion of a spread of planes of $P G(5,2)$ is, in the threequbit case, intimately related to the very important notion of mutually unbiased bases.

For the sake of completeness, we will also present the $8 \times 8$ representation of the remaining generator of $\operatorname{Sp}(6,2), \beta$, which is of order two. In order to calculate the relevant matrix, we rewrite the $6 \times 6$ symplectic matrix given by eq. (3.16) in a block form consisting of $3 \times 3$ matrices $a, b, c, d$ as

$$
D(\beta)=\left(\begin{array}{ll}
a & b \\
c & d
\end{array}\right),
$$

where the individual blocks can be readily read off from eq. (3.16). Then, under the transformation $(A \mid B) \mapsto(A a+B c \mid A b+B d)$, we get

$$
\begin{aligned}
m^{\prime} & =\operatorname{Det}(A a+B c)=\operatorname{Det}(A a)+\operatorname{Tr}\left(A a c^{\sharp} B^{\sharp}\right)+\operatorname{Tr}\left(B c a^{\sharp} A^{\sharp}\right)+\operatorname{Det}(B c) \\
& =\operatorname{Det} A+\operatorname{Tr}\left(c a^{\sharp} A^{\sharp} B\right)=m+\operatorname{Tr}\left(c a^{\sharp} N\right),
\end{aligned}
$$


where we have taken into account that, according to eq. (3.16), $\operatorname{Det} a=1$, Det $c=0$, and $c^{\sharp}=0$, and also employed definition (4.14). Using the explicit forms of the matrices $a$ and $c$ as well as expressions (4.11), one finds

$$
\tilde{P}_{123}=P_{123}+P_{234}+P_{135} .
$$

Similar manipulations yield the transformation laws

$$
\begin{array}{lll}
\tilde{P}_{456}=P_{456}+P_{345}, & & \\
\tilde{P}_{156}=P_{156}+P_{135}+P_{456}, & \tilde{P}_{246}=P_{246}+P_{234}+P_{456}, & \tilde{P}_{345}=P_{345}, \\
\tilde{P}_{234}=P_{234}+P_{345}, & \tilde{P}_{135}=P_{135}+P_{345}, & \tilde{P}_{126}=P_{126}+P_{246}+P_{156}+P_{123}
\end{array}
$$

hence, the corresponding matrix that acts on row vectors from the right has the form

$$
R(\beta)=\left(\begin{array}{llllllll}
1 & 0 & 0 & 0 & 0 & 0 & 0 & 1 \\
0 & 1 & 0 & 0 & 0 & 0 & 0 & 1 \\
0 & 0 & 1 & 0 & 0 & 0 & 0 & 1 \\
0 & 0 & 0 & 1 & 1 & 1 & 1 & 0 \\
0 & 1 & 1 & 0 & 1 & 0 & 0 & 0 \\
1 & 0 & 1 & 0 & 0 & 1 & 0 & 0 \\
1 & 1 & 0 & 0 & 0 & 0 & 1 & 0 \\
0 & 0 & 0 & 0 & 0 & 0 & 0 & 1
\end{array}\right) .
$$

One can verify that $R^{2}(\beta)=I_{8}$.

What we have constructed here is an explicit realization of the so-called spin module, or spin representation, of $\operatorname{Sp}(6,2)[21,22]$. In the mathematical literature, the 8-dimensional representation space for $\operatorname{Sp}(6,2)$ that corresponds to our $\mathcal{V}_{4}$ is constructed as a quotient space of the 14-dimensional space $\mathcal{V}_{7}$ with respect to the unique maximal subspace $\mathcal{V}_{3}$, fixed by $\operatorname{Sp}(6,2)$.

Finally, it is also worth noticing that the block-diagonal nature of $R(\alpha)$ corresponds to the fact that $D(\alpha)$ of eq. (3.13) gives a representation for one of the generators of $G L(3,2)=\mathrm{SL}(3,2)$, the latter being a subgroup of $\operatorname{Sp}(6,2)$ consisting of block-diagonal $6 \times 6$ matrices. Then using instead $K$ and $L$ of eq. (3.13) any two matrices, say $a$ and $d$, of $G L(3,2)$, related as $a^{\sharp}=d^{T}$, the corresponding action of an element of $\operatorname{SL}(3,2)$ on the fourqubit operators is just the usual action coming from the one that can be constructed on three-qubits. This means that the first entry of a four-qubit operator is left invariant, and the last three ones are transformed according to this particular three-qubit representation. Such a construction then trivially leads to an SL(3,2)-representation on the four-qubit counterparts of the elements of $\mathcal{I}$.

\section{Mermin's pentagrams}

Our formalism has now been developed to such an extent that it can be employed to gain fundamental insights into the structure of so-called Mermin's pentagrams, objects living inside our symplectic polar space $\mathcal{W}(5,2)$ and central to the conceptual issues related to 
quantum contextuality. Introduced by Mermin [11], a Mermin's pentagram is a configuration consisting of ten three-qubit operators arranged along five edges sharing pairwise a single point. Each edge features four operators that are pairwise commuting and whose product is $+I I I$ or $-I I I$, with the understanding that the latter possibility occurs an odd number of times. A recent computer search [10] has shown that $\mathcal{W}(5,2)$ contains altogether 12096 Mermin's pentagrams, of which 336 are formed of solely symmetric observables. It was also pointed out that these numbers are rather remarkable, since 12096 is the order of the group $G_{2}(2)$, which is the automorphism group of the smallest split Cayley hexagon, and 336 is just the twice of the order of $\mathrm{SL}(3,2)$, the latter being the automorphism group of the smallest projective plane. We shall, among other things, provide an elegant computer-free justification of the occurrence of the second number.

To begin with, one recalls [8] that an edge of a pentagram represents an affine plane of order two, i.e. the plane that originates from the Fano plane via omitting one of its lines. Now, as each Fano plane gives birth to seven such affine planes and $\mathcal{I}$ features 135 Fano planes, then we have altogether 945 copies of affine planes, each a possible candidate for an edge of a Mermin's pentagram. In this pool of affine planes we will look for such quintuples that have the above-described intersection property; every such quintuples will thus be a potential candidate for a Mermin's pentagram.

To this end in view, we will first have a look at the set of 30 planes that are lying on our particular Klein quadric $\mathcal{Q}$, accommodating all symmetric three-qubit observables. As already described (see section 3), these planes form two distinct systems of cardinality 15 each. One system (let us call it $\mathcal{L}$ ) consists of

$$
\begin{aligned}
&\{X I I, X Y Y, I Y Y, X Z X, I X Z, X X Z, I Z X\} \leftrightarrow X X I I, \\
&\{X X X, Z X Z, Y I Y, X Z Z, Y Y I, Z Z X, I Y Y\} \leftrightarrow X X X X, \\
&\{I X I, X X Z, X I Z, Y X Y, Z I X, Z X X, Y I Y\} \leftrightarrow X I X I, \\
&\{X X I, Z Z X, Y Y X, I X Z, Z Y Y, Y Z Y, X I Z\} \leftrightarrow X X X I, \\
&\{I I X, Z X X, Z X I, Y Y I, X Z X, X Z I, Y Y X\} \leftrightarrow X I I X, \\
&\{I X X, Y Z Y, Y Y Z, Z I X, X Z Z, X Y Y, Z X I\} \leftrightarrow X I X X, \\
&\{X I X, X Z I, I Z X, Z Y Y, Y X Y, Z X Z, Y Y Z\} \leftrightarrow X X I X, \\
&\{I I X, Z I I, I Z I, Z Z I, Z I X, I Z X, Z Z X\} \leftrightarrow I I I X, \\
&\{I X X, Z Z Z, I Z Z, Z I I, Z Y Y, I Y Y, Z X X\} \leftrightarrow I I X X, \\
&\{X I X, I Z I, Z I Z, Z Z Z, X Z X, Y I Y, Y Z Y\} \leftrightarrow I X I X, \\
&\{X I I, I Z Z, I I Z, I Z I, X Z Z, X I Z, X Z I\} \leftrightarrow I X I I, \\
&\{X X X, Z I Z, Z Z I, I Z Z, Y X Y, Y Y X, X Y Y\} \leftrightarrow I X X X, \\
&\{I X I, I I Z, Z I I, Z I Z, I X Z, Z X I, Z X Z\} \leftrightarrow I I X I, \\
&\{X X I, Z Z I, Z Z Z, I I Z, Y Y I, Y Y Z, X X Z\} \leftrightarrow I X X I, \\
&\{X X I, I I X, I X X, X I X, X I I, X X X, I X I\} \leftrightarrow X I I I .
\end{aligned}
$$

The 15 planes of the other system $($ called $\mathcal{G}$ ) are those that feature swapped entries $Z$ and $X$ in labeling of both three- and four-qubit operators. Notice that for each plane 
from (6.1)-(6.14) the last three entries of the four-qubit label are identical with the threequbit label of the first element. One can see that all the 15 planes share pairwise a single point and the corresponding four-qubit operators are pairwise commuting. It is worth pointing out here that the special plane

$$
\{Z I I, Z Z Z, I Z I, I Z Z, Z I Z, I I Z, Z Z I\} \leftrightarrow Z I I I
$$

is disjoint from the planes defined by eqs. (6.1)-(6.7) and eq. (6.15), since the corresponding four-qubit operators anticommute, and sharing a line with each of the planes (6.8)-(6.14), where the corresponding four-qubit representatives commute.

Let us now consider pentads of pairwise commuting four-qubit operators from $\mathcal{L}$ such that their product is IIII. A handy example is the set $\{X X X X, X I I I, I X I I, I I X I, I I I X\}$. One can readily see that the ten three-qubit operators coming from pairwise intersections of the corresponding planes form a pentagram,

$$
\begin{array}{ll}
\{X X X, Z Z X, Z X Z, X Z Z\}, & \{X X X, I I X, X I I, I X I\}, \quad\{X Z Z, I I Z, X I I, I Z I\}, \\
\{Z I I, Z Z X, I I X, I Z I\}, & \{Z X Z, I I Z, Z I I, I X X\} .
\end{array}
$$

Another illustrative example is $\{X I I I, X X X X, I X X I, I I X I, I I X X\}$, whose associated pentagram looks as follows

$$
\begin{array}{ll}
\{X X X, Y Y I, I Y Y, Z X Z\}, & \{X X X, X X I, I X I, I X X\}, \quad\{Z X Z, Z I I, I X I, I I Z\}, \\
\{Z Z Z, Y Y I, X X I, I I Z\}, & \{Z Z Z, I Y Y, Z I I, I X X\} .
\end{array}
$$

Notice that in both examples the four-qubit representatives of the planes giving birth to pentagrams satisfy not only the property $A B C D E=I I I I$, but also the constraint that no three of them are on a common line. Also, as the attentive reader might have noticed, in both examples the four-qubit observables feature only two different entries, namely $X$ and $I$. Our next task will be to find all the other cases of this type.

To this end, we express four-qubit labels in the form $X \otimes \mathcal{A}_{i}$ and $I \otimes \mathcal{A}_{i}$, where $\mathcal{A}_{i}, i=1,2, \cdots, 7$, are three-qubit operators that will be used to label the points of a Fano plane. The remaining operator $X \otimes I I I$ is taken to have a special footing. Let us first focus on such quadruples of operators $\left\{\mathcal{A}_{1}, \mathcal{A}_{2}, \mathcal{A}_{3}, \mathcal{A}_{4}\right\}$ that correspond to anti-flags of the Fano plane. An anti-flag consists of a line and a point not incident with that line; for example, the set $\{X X X, I I X, I X I, I X X\}$, where the point is represented by $X X X$ and the line by the triple $\{I I X, I X I, I X X\}$. There are 28 anti-flags in the Fano plane, each generating four pentagrams; hence, altogether 112 pentagrams of this kind. The four pentagrams coming from the above-given example are

$$
\begin{array}{llrrr}
I \otimes X X X, & X \otimes X X X, & X \otimes I X I, & X \otimes I I X, & X \otimes I X X, \\
I \otimes X X X, & X \otimes X X X, & X \otimes I X I, & I \otimes I I X, & I \otimes I X X, \\
I \otimes X X X, & X \otimes X X X, & I \otimes I X I, & X \otimes I I X, & I \otimes I X X, \\
I \otimes X X X, & X \otimes X X X, & I \otimes I X I, & I \otimes I I X, & X \otimes I X X .
\end{array}
$$


The next kind of a quadruple $\left\{\mathcal{A}_{1}, \mathcal{A}_{2}, \mathcal{A}_{3}, \mathcal{A}_{4}\right\}$ corresponds to the complement of a line of the Fano plane, i.e. to the point-set of the associated affine plane of order two, which we will refer to as a quadrangle. We have seven such quadrangles. An example is the set $\{X X X, X I I, I X I, I I X\}$, which is the complement of the line $\{X X I, X I X, I X X\}$. Obviously, this construction yields two classes of such pentagrams, and of cardinality 28 each, which amounts to 56 pentagrams of this kind. For our particular example, the fourqubit labels of these $4+4$ pentagrams are as follows

$$
\begin{array}{rrrrr}
I \otimes X X X, & X \otimes X I I, & X \otimes I I X, & X \otimes I X I, & X \otimes I I I, \\
X \otimes X X X, & I \otimes X I I, & I \otimes I X I, & I \otimes I I X, & X \otimes I I I,
\end{array}
$$

where the missing three pentagrams from each class arise via a cyclic shift of the operators $I$ and $X$ in the leftmost qubit to the remaining members of the three-qubit operators belonging to the quadrangle. Thus, for example, the next member of the class given by eq. (6.24) is $\{X X X X, I X I I, X I I X, X I X I, X I I I\}$. We thus arrive at the total of 168 pentagrams coming from the planes of system $\mathcal{L}$. Following the same procedure with $X$ replaced by $Z$, that is with the planes from the other system, $\mathcal{G}$, results in another set of 168 pentagrams. All in all, we find 336 pentagrams that can be formed from symmetric three-qubit observables. It represents no difficulty to verify that all these pentagrams are, in fact, Mermin's pentagrams. This is one of the major results found in [10] with the aid of a computer. Here, we have not only succeeded in furnishing a rigorous, computer-free explanation of this finding, but also shown that the whole set of "symmetric" pentagrams can be generated from merely six basic types, given by eqs. (6.20)-(6.25). Nay, noticing that the leftmost qubit must be associated with either two or four $X$ operators, this classification can further be reduced to just two kinds: namely, anti-flag and quadrangle ones. Notice in passing that the special planes are tied uniquely to the quadrangle kind.

An alternative explanation for the number 336 goes as follows. We have seen that all the planes needed for construction of these pentagrams lie on the Klein quadric, $\mathcal{Q}$, defined as the zero locus $Q_{0}(v)=\sum_{i}^{3} a_{i} b_{i}=0$, where $v=\left(a_{1} a_{2} a_{3} b_{1} b_{2} b_{3}\right) \in \mathbb{Z}_{2}^{6}$. Under a transformation of SL(3,2) of the form (3.13), with $K$ and $L$ being replaced by nonsingular matrices $A$ and $D$ related to each other as $A^{\sharp}=D^{T}$,

$$
Q_{0}=\mathbf{a b}^{T} \mapsto \mathbf{a} A(\mathbf{b} D)^{T}=\mathbf{a} A D^{T} \mathbf{b}^{T}=\mathbf{a b}^{T},
$$

which means that $\mathrm{SL}(3,2)$ leaves the Klein quadric invariant. On the other hand, the transformation swapping the systems $\mathcal{L}$ and $\mathcal{G}$ is the one with its $6 \times 6$ matrix representative being just the matrix $J$ of eq. (2.9). This transformation also leaves $Q_{0}$, and so the Klein quadric, invariant. One can actually prove that the group $\mathrm{SL}(3,2) \cdot 2$ just described is a maximal subgroup of $G_{2}(2)[40,44]$. Moreover, $\mathrm{SL}(3,2) \cdot 2$ also lies inside the orthogonal group of $\mathcal{Q}$, which is isomorphic to $O^{+}(6,2)$. The facts that $\mathrm{SL}(3,2) \cdot 2$ is maximal inside $G_{2}(2)$ and that $O^{+}(6,2)$ possesses no subgroup isomorphic to $G_{2}(2)$ mean that $\mathrm{SL}(3,2) \cdot 2$ is the full stabilizer of $\mathcal{Q}$ in $G_{2}(2)$. Now, $\operatorname{SL}(3,2)$ is just the stabilizer of the special planes (6.15) and (6.16) that we used in our construction of the 336 pentagrams. The group $\mathrm{SL}(3,2) \cdot 2$, of order 336 , then acts transitively on these planes by simply exchanging them. 
It can be shown [23] that this group also acts transitively on the set of planes (6.8)-(6.14), and separately on the set of planes (6.1)-(6.7). These facts strongly indicate that it should be possible to use the $2 \times 168=336$ elements of $\mathrm{SL}(3,2) \cdot 2$ to generate all the 336 pentagrams from the canonical one given by eq. (6.17) and relate this group-theoretical method to the above-described geometric construction.

Obviously, the remaining pentagrams, which feature also antisymmetric operators, can be generated by the repeated action of $\operatorname{Sp}(6,2)$. For example, one can act on the canonical pentagram, eq. (6.17), by $D(\beta)$ of eq. (3.16) and on the corresponding four-qubit operators by eq. (5.43), to obtain the pentagram

$$
\begin{array}{ll}
\{Z I I, I Z I, I I X, Z Z X\}, & \{Z Y X, Z Z Y, Z X Z, Z I I\}, \quad\{Y Z X, Z Z Y, I Z I, X Z Z\}, \\
\{X X X, Z X Z, X Z Z, Z Z X\}, & \{Z Y X, Y Z X, I I X, X X X\} .
\end{array}
$$

The four-qubit operators labeling the planes whose intersections yield this pentagram are $\{I I I X, Z Z X I, Z X Z I, X X X X, X Z Z I\}$. They again satisfy the identity $A B C D E=I I I I$ and no three of them are collinear. The five observables are, of course, symmetric and pairwise commuting. By using the action of $D(\alpha)$ and $D(\beta)$ of $\operatorname{Sp}(6,2)$, one can then generate new pentagrams. Alternatively, one can generate the same pentagrams via the corresponding action of the generators $R(\alpha)$ and $R(\beta)$ on the associated four-qubit operators. Notice, however, that since the four-qubit operators are symmetric, the spin representation $R$ of $\operatorname{Sp}(6,2)$ on these operators can be expressed as a conjugate action of type (3.3). Accordingly, the condition $A B C D E=I I I I$ is preserved and, due to the symplectic nature of these transformations, the remaining constraints on the five four-qubit observables are left intact as well.

Can our approach also account for the total number of Mermin's pentagrams amounting to 12096, i.e. the order of $G_{2}(2)$ ? The authors of [10] made an intriguing conjecture that this number should stem form the properties of a remarkable point-line incidence geometry called the split Cayley hexagon of order two [37, 38], which has $G_{2}(2)$ as its automorphism group. Here, we can merely offer some remarks and conjectures on this issue, the details of which we would like to postpone to a separate paper. First of all, notice that $G_{2}(2)$ is a maximal subgroup of $\operatorname{Sp}(6,2)$. A useful presentation for this group convenient for our purposes is (see footnote 2 on page 9 )

$$
G_{2}(2)=\langle\alpha, \gamma\rangle, \quad \gamma=\beta \alpha^{2} \beta \alpha \beta \alpha^{3} \beta \alpha^{4} \beta,
$$

where $\beta$ corresponds to the transvection whose representative is given by $D(\beta)$ of eq. (3.16) and $\alpha$ is the usual cyclic shift generating the automorphism of order seven. Then, ${ }^{3}$

$$
D(\gamma)=\left(\begin{array}{llllll}
0 & 0 & 0 & 0 & 1 & 0 \\
0 & 0 & 1 & 1 & 1 & 0 \\
1 & 0 & 0 & 1 & 0 & 1 \\
1 & 1 & 0 & 1 & 0 & 0 \\
1 & 0 & 1 & 0 & 1 & 0 \\
0 & 0 & 1 & 0 & 0 & 0
\end{array}\right), \quad R(\gamma)=\left(\begin{array}{llllllll}
0 & 0 & 0 & 1 & 1 & 0 & 1 & 0 \\
0 & 0 & 0 & 0 & 0 & 0 & 1 & 0 \\
0 & 0 & 0 & 1 & 0 & 1 & 1 & 0 \\
1 & 1 & 0 & 0 & 1 & 1 & 0 & 1 \\
1 & 0 & 0 & 1 & 0 & 0 & 1 & 0 \\
1 & 1 & 1 & 0 & 1 & 1 & 0 & 0 \\
0 & 1 & 0 & 1 & 0 & 0 & 1 & 0 \\
0 & 0 & 0 & 1 & 0 & 0 & 0 & 0
\end{array}\right) .
$$

\footnotetext{
${ }^{3}$ We thank Zsolt Szabo for checking these matrices for us on a computer.
} 
These matrices are of order six. The matrix $R(\gamma)$ leaves invariant the special antisymmetric four-qubit operator YIII. Since, according to eq. (5.32), the other generator $R(\alpha)$ operates exclusively on the last three qubits via a cyclic shift and leaves $I I I$ invariant, $R(\alpha)$ and $R(\gamma)$ generate a maximal $G_{2}(2)$ subgroup of $\operatorname{Sp}(6,2)$, leaving $Y I I I$ invariant. This conforms to a theorem [39] that states that if we have a point lying off the $\mathcal{Q}^{+}(7,2)$, then its stabilizer within $\operatorname{Sp}(6,2)$ is isomorphic to $G_{2}(2)$, and there is a single conjugacy class of $G_{2}(2)$ 's in $\operatorname{Sp}(6,2)$. In our language of four-qubit observables this means that the stabilizer of each antisymmetric operator (which is not an element of our quadric accommodating only symmetric ones) defines a $G_{2}(2)$ subgroup. Since we have 120 antisymmetric four-qubit operators, there are 120 possibilities for obtaining a $G_{2}(2)$ subgroup of $\operatorname{Sp}(6,2)$. This, clearly, reflects the fact that $|\operatorname{Sp}(6,2)| /\left|G_{2}(2)\right|=1451520 / 12096=120$.

A point of the four-qubit symplectic polar space, $\mathcal{W}(7,2)$, is collinear with 126 other points of this space (see, for example, [5] and references therein). If this point lies off the quadric $\mathcal{Q}^{+}(7,2)$, then 63 of these points will be located on the quadric itself, being at the same time the points of a copy of the split Cayley hexagon of order two. Let us now consider a particular set of 63 operators, each of which commutes with the special (antisymmetric) operator $Y I I I$ :

$$
Y \otimes \mathcal{A}, \quad \mathcal{A}^{T}=-\mathcal{A}, \quad I \otimes \mathcal{S}, \quad \mathcal{S}^{T}=\mathcal{S}
$$

Here, $\mathcal{A}$ represents the set of 28 antisymmetric and $\mathcal{S}$ stands for the set of 35 nontrivial symmetric three-qubit operators. Now, if one disregards the first-qubit labels, these 63 operators can be viewed as the 63 non-trivial elements of the three-qubit Pauli group, which were employed by two of us [14] to label a copy of the split Cayley hexagon of order two when embedded in the corresponding three-qubit polar space $\mathcal{W}(5,2)$. A diagrammatic illustration of the structure of our hexagon, together with the corresponding labeling, is shown in figure 1 . Hence, as a representative of the hexagon living inside $\mathcal{Q}^{+}(7,2)$ one can use the pictorial representation of figure 1 , with the only difference being that, according to (6.30), one has to also include the extra labels $Y$ and $I$ of the extra qubit. Then the $G_{2}(2)$ action on the points and lines of this particular representation of the split Cayley hexagon of order two is generated by $R(\alpha)$ and $R(\gamma)$.

Having at our disposal an explicit form of the bijective correspondence between the points of $\mathcal{Q}^{+}(7,2)$ and the planes of $\mathcal{I}$ (section 5), one can address the following interesting question: what kind of triples of planes are the lines of the hexagon mapped to? According to Theorem 3.4 of [23], the lines of $\mathcal{Q}^{+}(7,2)$ are of two types; a line of one type arises from a pencil of planes of $\mathcal{I}$, whereas that of other type comes from a plane-star on a fixed quadric. For example, a plane-star on our particular Klein quadric is any set of three planes in the same system $(\mathcal{L}$ or $\mathcal{G})$ that share a point, that is, any triple of planes from eqs. (6.1)-(6.15) such that when their representative four-qubit operators are multiplied, the result is $I I I I$. As an example, one can take the planes defined by eqs. (6.1), (6.2) and (6.9), labeled by $X X I I, X X X X$ and $I I X X$, whose common point corresponds to $I Y Y$. A line arising from a pencil of planes is, for example, the $\{I X I X, I I Z I, I X Z X\}$ 


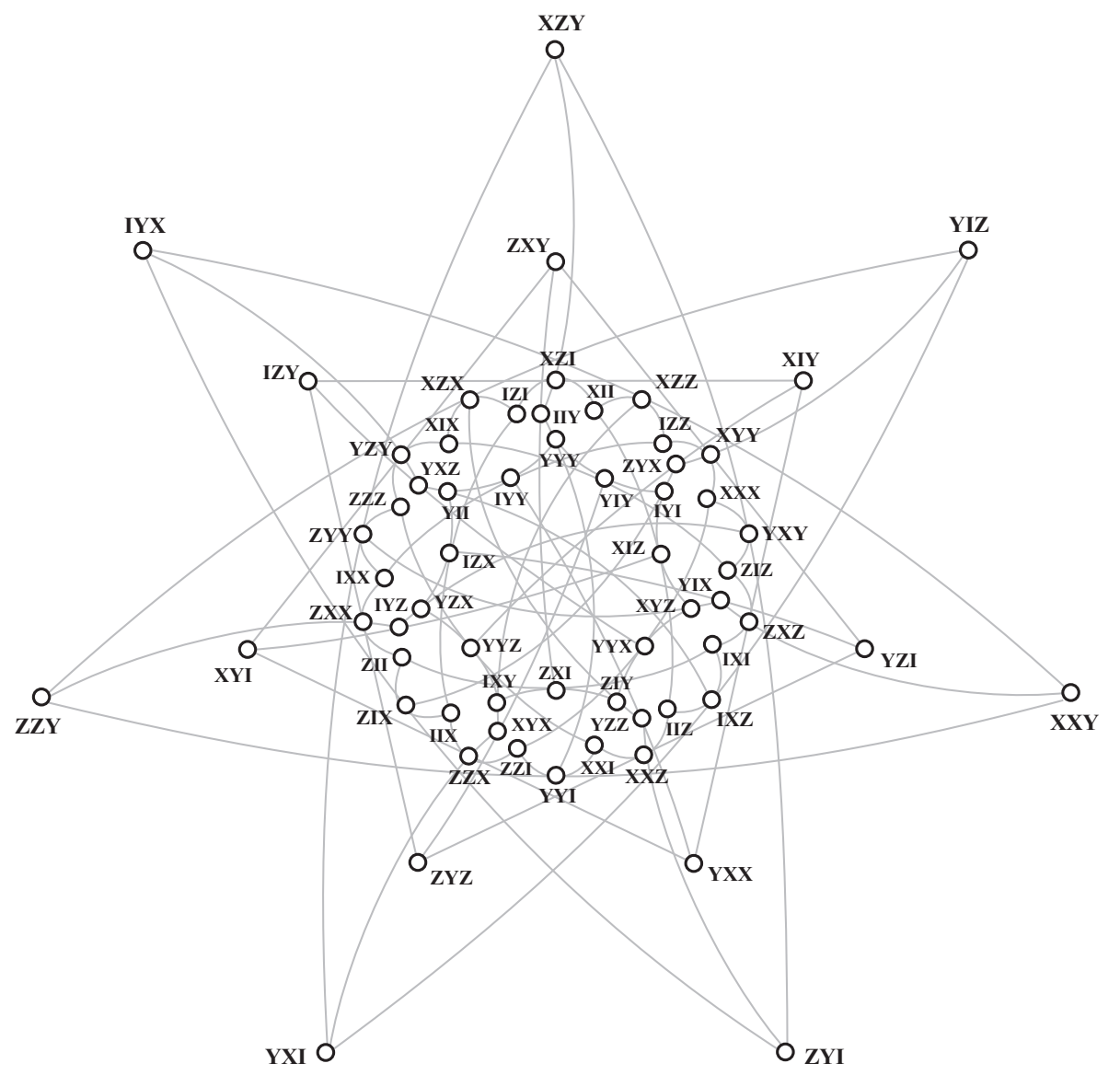

Figure 1. A diagrammatic illustration of the structure of the split Cayley hexagon of order two (based on drawings given in $[37,38]$ ). The points are illustrated by small circles and its lines by triples of points lying on the same segments of straight-lines and/or arcs. Labeling by the elements of $\mathcal{P}_{3}$ is adopted from [14]. Also obvious is an automorphism of order seven of the structure.

one, as its corresponding planes

$$
\begin{aligned}
& \{X I X, I Z I, Z I Z, Z Z Z, X Z X, Y I Y, Y Z Y\}, \\
& \{I Z I, I I X, X I I, X I X, I Z X, X Z I, X Z X\}, \\
& \{Y Z Z, Z Z Y, Z I Y, Y I Z, X I X, X Z X, I Z I\},
\end{aligned}
$$

share indeed a line, namely the $\{X I X, X Z X, I Z I\}$ one. ${ }^{4}$ A closer look at figure 1 reveals that this line also belongs to our hexagon. Employing the formalism of [14] and Theorem 4.1 of [23], it can be verified that all the lines of our split Cayley hexagon are of this "pencilof-planes" type.

So, then, how is the aggregate of Mermin's pentagrams related to the split Cayley hexagon of order two and its automorphism group $G_{2}(2)$ ? Clearly, the above considerations

\footnotetext{
${ }^{4}$ It is interesting to note that the coordinates of this line are exactly those of the associated four-qubit operators with the first-qubit entry omitted.
} 
imply that out of the five planes generating a Mermin's pentagram, no three can be in a pencil of planes that corresponds to a line of the hexagon. Moreover, as our hexagon picks up only 63 planes from $\mathcal{I}$, these particular planes should somehow be used as a core set for labeling the totality of pentagrams with elements of $G_{2}(2)$. An investigation along these lines is under way and will be the subject of a separate paper.

\section{A link with the Black-Hole-Qubit correspondence}

String/ $M$-theory is the theory of extended objects, membranes and strings. As it is well known, dynamics of such objects can consistently be described provided that the ambient space-time has extra-dimensions. There exist different types of consistent string theories, connected to each other by symmetries, called duality symmetries [16]. In the low-energy limit, these string theories give rise to effective low-energy supersymmetric field theories. When compactifying the low-energy effective actions, these extra-dimensions are curled up into tiny compact spaces, and one is left with the usual four-dimensional "macroscopic" space-time. Under the process of curling up of the extra-dimensions, the wrapping configurations of extended objects on nontrivial submanifolds of the compact space manifest themselves via the occurrence of charges, of both magnetic and electric type. There are also special scalar fields originating from this mechanism, called moduli fields. They come from fields describing the volume and shape of the extra dimensions. The charges and moduli might form special configurations that can give rise to special space-time curvature effects, yielding charged extremal black holes in four dimensions. There can be both supersymmetric and non-supersymmetric black holes. In the case of toroidal compactifications, when the compact extra-dimensions are tiny tori of six dimensions for string- and seven dimensions for $M$-theory, the resulting four-dimensional theory is called $N=8$ supergravity.

It is also a well-known fact that the most general class of charged, extremal blackhole solutions in $N=8$ supergravity/ $M$-theory in four dimensions is characterized by 56 charges [16], equally-splitted into electric and magnetic ones. These black-hole solutions are the ones of the classical equations of motion of $N=8$ supergravity exhibiting an $E_{7(7)}$ symmetry, where $E_{7(7)}$ is the non-compact real form of the exceptional group $E_{7}$ with the 56 charges transforming according to its fundamental irreducible representation. The corresponding black-hole solutions also display this symmetry via their semiclassical Bekenstein-Hawking entropy formulas, which are quartic polynomials invariant under $E_{7(7)}$. At the level of quantum theory, the charges will be quantized and the symmetry group will be the discrete subgroup $E_{7}(\mathbb{Z})$, called the $U$-duality group. An important subgroup of this group is $W\left(E_{7}\right)$. This Weyl group can be regarded as the generalization of the usual group of electric -magnetic duality, known from classical electrodynamics [20].

As already stressed, $W\left(E_{7}\right)=\operatorname{Sp}(6,2) / \mathbb{Z}_{2}$ and since $\operatorname{Sp}(6,2)$ has been shown to be intimately related to three-qubit observables, one may suspect that the structure of the black-hole entropy and the 56-dimensional fundamental representation of $E_{7}$ can both be given a three-qubit-based reinterpretation. This is indeed the case. The relevant reinterpretation can be presented within a theoretical framework based on the tripartite entanglement of seven qubits $[17,30]$. The main idea is that $E_{7}$, as a group of rank seven, 
contains seven copies of the rank-one groups SL(2). In quantum information, $\mathrm{SL}(2)$ is the group of admissible local manipulations of a qubit [29]; this is the group of stochastic local operations and classical communication (SLOCC). Next, the fundamental $7 \times 8=56$ dimensional irrep of $E_{7}$ can be decomposed into seven copies of the 8-dimensional threequbit Hilbert spaces according to a nice pattern dictated by the incidence structure of the Fano plane $[13,14,17,19,30]$. A similar seven-qubit based understanding of the Liealgebra of $E_{7}$ via the 133-dimensional adjoint representation is also possible [17, 41, 42]. Hence, within the context of the BHQC, a clear understanding of possible patterns of $\mathrm{SL}(2)$-subgroups isomorphic to $\mathrm{SL}(2)^{7}$ of the $E_{7}$ is of utmost importance. This task has been carried out by Cerchiai and van Geemen [19]. Here, we would like to reiterate the basic idea of this work by showing its connection to the structure of our context space $\mathcal{I}$.

The basic observation of [19] is that the root lattice of $E_{7}, L\left(E_{7}\right)$, defined as

$$
L\left(E_{7}\right) \equiv\left\{l_{1} \alpha_{1}+\cdots+l_{7} \alpha_{7} \mid l_{a} \in \mathbb{Z}\right\}
$$

with $\alpha_{a}, a=1,2, \cdots, 7$, being the simple roots of $E_{7}$, can be mapped into our vector space $V_{3} \simeq \mathbb{Z}_{2}^{6}$ as follows

$$
\pi: L\left(E_{7}\right) \rightarrow V_{3}, \quad \pi\left(l_{1} \alpha_{1}+\cdots+l_{7} \alpha_{7}\right)=l_{1} v_{1}+\cdots+l_{7} v_{7},
$$

where the numbers $l_{a}$ on the right-hand side are to be understood $\bmod 2$, and where the details of the correspondence between the simple roots $\alpha_{a}$, labeling the nodes of the Dynkin diagram of $E_{7}$, and certain three-qubit observables, $v_{a}$, can be found in [19]. Here, we only note that the core of this correspondence is the relation

$$
\left(\alpha_{a}, \alpha_{b}\right)=\left\langle v_{a}, v_{b}\right\rangle \bmod 2
$$

which establishes a relation between the inner product of the root system on the left-hand side and our symplectic product given by eq. (2.4) on the right-hand side. Making use of $\pi$, one can map the 126 roots of $E_{7}$ to the 63 nonzero elements of $V_{3}$. Note that $\pi(\alpha)=$ $\pi(-\alpha)$, and that the Weyl reflections in the root system correspond to the transvections of eq. (2.11). Since the Weyl reflections generate $W\left(E_{7}\right)$ and the transvections generate $\operatorname{Sp}(6,2)$, the map $\pi$ establishes the already-mentioned isomorphism $W\left(E_{7}\right) / \mathbb{Z}_{2} \simeq \operatorname{Sp}(6,2)$.

A positive root $\alpha$ induces an $s l(2)$-subalgebra with standard generators $\left\{X_{\alpha}, X_{-\alpha}, H_{\alpha}\right\}$, where $H_{\alpha}=\left[X_{\alpha}, X_{-\alpha}\right]$ lies within the seven-dimensional Cartan subalgebra of $e_{7}$. One can then show [19] that the generators of the subalgebras $s l_{\alpha}(2)$ and $s l_{\beta}(2)$, determined by two different positive roots $\alpha$ and $\beta$, commute if, and only if, these roots are orthogonal. By virtue of eq. (7.3), this means that two commuting three-qubit observables can be associated with two commuting copies of $s l(2)$-algebras in $e_{7}$, i.e. with the SLOCC-algebras of two distinguishable qubits. Since $E_{7}$ is of rank seven, its root system spans $\mathbb{R}^{7}$; hence, there are no more than seven mutually commuting orthogonal roots. Using the map $\pi$, this corresponds to the fact that the maximum number of pairwise commuting three-qubit observables is seven (our heptad). Hence, the set of maximum sets of mutually orthogonal roots in the root system of $E_{7}$ has the same structure as our context space $\mathcal{I}$. Moreover, since mutually orthogonal systems of roots correspond to an assignment of seven qubits with their seven commuting $s l(2)$ SLOCC-algebras, this establishes a correspondence between our method(s) of 
studying $\mathcal{I}$ and the seven-qubit picture of the BHQC. Indeed, as there are 135 maximum sets of mutually orthogonal roots, there are also 135 root subsystems $\mathrm{SL}(2)^{\oplus 7} \subset E_{7}$ that can give rise to sets of seven-qubit systems occurring in the BHQC framework.

As a second application lying within the realm of the physics of black holes in string theory, we will show that mapping sets of three-qubit operators to a special subset of fourqubit operators might even be relevant for shedding some new light on issues of dimensional reduction within a finite geometric framework. Since the main incentive of this paper is merely to set the finite geometric ground for further applications in connection with the BHQC, let us just present here several rudimentary observations forming the basis of this interesting correspondence. The detailed elaboration of these ideas will be postponed to a future publication.

We have already mentioned at the beginning of this section that the scalar fields play a crucial role in determining the structure of static extremal black hole solutions of effective four-dimensional supergravities. Mathematically, these fields live in a symmetric space of the form $G_{4} / H_{4}$, where $G_{4}$ is the four-dimensional $U$-duality group and $H_{4}$ its maximal compact subgroup. In the case of maximal $N=8$ supergravity, we have $G_{4}=$ $E_{7(7)}$ and $H_{4}=\mathrm{SU}(8)$. Working in the $\mathrm{SU}(8)$-basis, the central charge that shows up in the $E_{7(7)}$-symmetric black-hole entropy formula is a complex antisymmetric $8 \times 8$ matrix; this matrix can be expanded in terms of the 28 antisymmetric three-qubit operators as basis vectors, where the 56 charges are displayed as the complex expansion coefficients. In this context, the relevant finite geometric structures have already been discussed in detail $[14,15]$. Moreover, the structure of the $E_{7(7)}$-symmetric black-hole entropy formula can alternatively be described by the finite geometry based on a particular graph studied by Cooperstein, which is closely related to the $E_{7}$-Gosset polytope [43, 44].

However, we can go even one step further and try to understand stationary black-hole solutions. It is well known that such solutions can effectively be studied by performing a time-like dimensional reduction of the corresponding four-dimensional supergravities, the result being a three-dimensional gravity coupled to new scalars featuring a nonlinear sigma model $[45,46]$. These new scalar fields that contain as a subset the original ones now form a pseudo-Riemannian symmetric space $G_{3} / H_{3}^{*}$, with the line-element given by a pseudoRiemannian metric. Here, $G_{3}$ is the three-dimensional $U$-duality group and $H_{3}^{*}$ is the maximally non-compact real form of $H_{3}(\mathbb{C})$, the complexification of the maximal compact subgroup $H_{3} \subset G_{3}$. In our special case, $G_{3}=E_{8(8)}$ and $H_{3}^{*}=S O^{*}(16)$. It can be shown that in this picture extremal black-hole solutions of the original four-dimensional theory can be mapped to the null geodesics on the $\operatorname{coset} G_{3} / H_{3}^{*}$ and are classified in terms of the adjoint orbits under $G_{3}$ of a nilpotent $G_{3}$-Lie-algebra-valued conserved Noether charge $Q$ [47, 48]. In addition to the usual electric and magnetic charges (amenable to a finite geometric interpretation based on three-qubit operators), $Q$ also contains the NUT-charge and other conserved charges corresponding to four-dimensional duality rotations [49]. Hence, an attempt to understand these new quantities in a finite geometric setting based on some $N$-qubit system, with $N>3$, is also very appealing.

Given our example of maximal supergravity, one can readily characterize the $E_{8(8)}$-Liealgebra-valued Noether charge in an $S O^{*}(16)$-basis defined in terms of $16 \times 16$ matrices. As 
the number of generators of this group is 120, the relevant object serving as a suitable basis in this setting is a specific set of 120 four-qubit operators. Its associated finite geometric structure can be found as follows. The group $S O^{*}(16)$ is the matrix group of $16 \times 16$ matrices $g$ satisfying

$$
g^{t} g=1, \quad g^{\dagger} \omega g=\omega, \quad \omega=Y \otimes I \otimes I \otimes I .
$$

Writing $g=\mathbf{1}+\mathcal{Z}+\ldots$, the above conditions yield

$$
\mathcal{Z}^{t}=-\mathcal{Z}, \quad \mathcal{Z}^{\dagger} \omega=-\omega \mathcal{Z}
$$

The latter equation implies that $\omega \mathcal{Z}$ is a Hermitian matrix, whereas the former one leads to some extra constraints

$$
\omega \mathcal{Z}=S+i A, \quad S^{t}=S, \quad S \omega=\omega S, \quad A^{t}=-A, \quad A \omega=-\omega A .
$$

Let us now consider the quadratic form $Q_{w}$ (cf. eq. (2.16)) associated with the element $w=(10001000) \in V_{4}$ representing the four-qubit operator $\omega$. Using the definition of $Q_{w}$ one can see that the points $v \in V_{4}$ of the elliptic quadric $\mathcal{Q}^{-}(7,2)$ given by the equation $Q_{w}(v)=0$ correspond to a set of 119 four-qubit operators. According to eq. (2.16), this set splits into 63 symmetric operators that commute and 56 antisymmetric ones that anticommute with $\omega$. Omitting the identity, these operators are precisely the ones occurring in the expansion of $\omega \mathcal{Z}$ (7.6), with $\mathcal{Z}$ being an element of the Lie-algebra of $S O^{*}(16)$. Again disregarding the identity operator, this expansion features altogether 63 symmetric fourqubit operators that lie on our quadric $\mathcal{Q}^{+}(7,2)$ (see section 2). It is easy to see that these 63 operators are precisely the ones defined by eq. (6.30), giving rise to the points of a copy of our split Cayley hexagon. Hence, the finite geometric structures studied in this paper occur naturally in this context of time-like dimensional reduction.

On the other hand, the 120 four-qubit operators contained in the expansion of $\omega \mathcal{Z}$ operators can be used to label the vertices of the projective version of the $E_{8}$ Gosset polytope [50]. This is a finite geometric object on which the Weyl group of $E_{8}-$ a discrete subgroup of the three dimensional $U$-duality group $G_{3}$ responsible for the 3Dversion of electric-magnetic duality - acts naturally. Moreover, the $E_{8}$-polytope contains as the vertex figure the $E_{7}$ Gosset polytope. In a natural labelling of the vertices of the $E_{8}$-polytope in terms of four-qubit operators, this $E_{7}$-polytope is labelled by merely threequbit ones. This subset, as expected, gives back the graph of Cooperstein encapsulating the structure of the $E_{7(7)^{-}}$symmetric black-hole entropy formula [43].

To briefly recapitulate, within the framework of the maximal $N=8$ supergravity, a dimensional reduction from four to three dimensions can be characterized by particular mappings of three-qubit operators and their associated finite geometric structures to certain sets of four-qubit operators with their corresponding finite geometric objects. These mappings and allied structures can possibly provide a finite geometric way for understanding the structure of the Noether charge $Q$ and, via the nilpotent orbits of $Q$, also of the different classes of the extremal black-hole solutions. 
We mention in closing that the physical meaning of the extra qubit showing up under dimensional reduction can nicely be identified in the so-called STU truncation [51] of $N=8$ supergravity, where the extra SL(2) group acting on the extra qubit corresponds to the Ehlers group $[52,53]$ well known to general relativists. A similar identification of physical quantities on the black-hole side with the ones on the finite-geometric side is envisaged. In a future work, we will hopefully be able to provide the reader with a more mature and elaborated form of this correspondence.

\section{Conclusions}

We have gained substantial insights into a yet-unnoticed relation between the three-qubit and four-qubit generalized Pauli groups, based on the so-called spin-module of the symplectic group $\operatorname{Sp}(6,2)$. Our starting point was the set $\mathcal{I}$ of 135 heptads of pairwise commuting three-qubit observables. We first labeled the elements of this distinguished subspace of $\mathcal{W}(5,2)$ by those of a seven-dimensional Clifford algebra. Then, by employing the formalism of Plücker/Grassmann embeddings, we worked out an explicit form of the bijection between $\mathcal{I}$ and the set of 135 symmetric four-qubit observables, lying on a particular hyperbolic quadric of $\mathcal{W}(7,2)$. After performing a detailed analysis of the action of $\operatorname{Sp}(6,2)$ on both sides of this correspondence, we gave a couple of interesting physical applications of our formalism. The first application concerned the structure of the set of 12096 Mermin's pentagrams living in $\mathcal{W}(5,2)$, as recently discovered with the aid of a computer [10]. Here, we have not only succeeded in furnishing a rigorous, computer-free explanation why there exist just 336 such pentagrams formed from the symmetric three-qubit observables, but also shown that the whole set of these "symmetric" pentagrams can be generated from merely six basic types (see eqs. (6.20)-(6.25)). Moreover, we also offered some hints linked with the structure of the split Cayley hexagon of order two - towards accounting for the number 12096 as well. Our second, BHQC, application made use of the fact that $\operatorname{Sp}(6,2)$ is related to the Weyl-group of $E_{7}$, which is a subgroup of the $U$-duality group responsible for electric-magnetic duality, which thus enabled us to reveal the relevance of our formalism for the corresponding BHQC framework.

\section{Acknowledgments}

A major part of this research was conducted within the "Research in Pairs" program of the Mathematisches Forschungsinstitut Oberwolfach (Oberwolfach, Germany), in the period from 24 February to 16 March, 2013. PL would also like to acknowledge financial support he received from the MTA-BME Condensed Matter Physics Research Group, grant No. 04119. MS was also partially supported by the VEGA Grant Agency, grant No.2/0003/13. We also thank the anonymous referee for a number of constructive remarks.

Open Access. This article is distributed under the terms of the Creative Commons Attribution License which permits any use, distribution and reproduction in any medium, provided the original author(s) and source are credited. 


\section{References}

[1] M.A. Nielsen and I.L. Chuang, Quantum Computation and Quantum Information, Cambridge University Press, Cambridge U.K. (2000).

[2] D. Gottesman, A class of quantum error correcting codes saturating the quantum Hamming bound, Phys. Rev. A 54 (1996) 1862 [quant-ph/9604038] [INSPIRE].

[3] D. Gottesman, A theory of fault tolerant quantum computation, Phys. Rev. A 57 (1998) 127 [quant-ph/9702029] [INSPIRE].

[4] A. Calderbank, E. Rains, N. Sloane and P. Shor, Quantum error correction and orthogonal geometry, Phys. Rev. Lett. 78 (1997) 405 [quant-ph/9605005] [INSPIRE].

[5] M. Saniga and M. Planat, Multiple qubits as symplectic polar spaces of order two, Adv. Studies Theor. Phys. 1 (2007) 1.

[6] H. Havlicek, B. Odehnal and M. Saniga, Factor-Group-Generated Polar Spaces and (Multi-)Qudits, SIGMA 5 (2009) 096.

[7] K. Thas, The geometry of generalized Pauli operators of $N$-qudit Hilbert space, and an application to MUBs, Europhys. Lett. 86 (2009) 60005.

[8] M. Saniga and P. Lévay, Mermin's pentagram as an ovoid of PG(3,2), Europhys. Lett. 97 (2012) 50006.

[9] M. Saniga, M. Planat, P. Pracna and P. Lévay, 'Magic' Configurations of Three-Qubit Observables and Geometric Hyperplanes of the Smallest Split Cayley Hexagon, SIGMA 8 (2012) 083.

[10] M. Planat, M. Saniga and F. Holweck, Distinguished three-qubit 'magicity' via automorphisms of the split Cayley hexagon, Quant. Inf. Process. 12 (2013) 2535.

[11] N.D. Mermin, Hidden variables and the two theorems of John Bell, Rev. Mod. Phys. 65 (1993) 803 [InSPIRE].

[12] N.D. Mermin, Simple unified form for the major no-hidden-variables theorems, Phys. Rev. Lett. 65 (1990) 3373.

[13] L. Borsten, M. J. Duff and P. Lévay, The black-hole/qubit correspondence: an up-to-date review, Class. Quant. Grav. 29 (2012) 224008 [arXiv:1206.3166].

[14] P. Levay, M. Saniga and P. Vrana, Three-Qubit Operators, the Split Cayley Hexagon of Order Two and Black Holes, Phys. Rev. D 78 (2008) 124022 [arXiv:0808.3849] [INSPIRE].

[15] P. Levay, M. Saniga, P. Vrana and P. Pracna, Black Hole Entropy and Finite Geometry, Phys. Rev. D 79 (2009) 084036 [arXiv:0903.0541] [InSPIRE].

[16] K. Becker, M. Becker and J. H Schwarz, String Theory and M-Theory: A Modern Introduction, Cambridge University Press, Cambridge U.K. (2006).

[17] P. Lévay, Strings, black holes, the tripartite entanglement of seven qubits and the Fano plane, Phys. Rev. D 75 (2007) 024024 [hep-th/0610314].

[18] M. Planat and P. Solé, Clifford groups of quantum gates, BN-pairs and smooth cubic surfaces, J. Phys. A 42 (2009) 042003.

[19] B.L. Cerchiai and B. van Geemen, From qubits to Er, J. Math. Phys. 51 (2010) 122203 [arXiv: 1003.4255] [INSPIRE]. 
[20] N. Obers and B. Pioline, U duality and M-theory, Phys. Rept. 318 (1999) 113 [hep-th/9809039] [INSPIRE].

[21] A. Cossidente and O.H. King, On twisted tensor product group embeddings and the spin representation of symplectic groups, Adv. Geom. 7 (2007) 55.

[22] R. Gow, Contraction of exterior powers in characteristic 2 and the spin module, Geom. Dedicata 64 (1997) 283.

[23] A. Cossidente and O. H. King, On the geometry of the exceptional group $G_{2}(q), q$ even, Des. Codes Cryptogr. 47 (2008) 145.

[24] J.W.P. Hirschfeld, Finite Projective Spaces of Three Dimensions, Clarendon, Oxford U.K. (1985).

[25] R. Penrose, Twistor algebra, J. Math. Phys. 8 (1967) 345 [InSPIRE].

[26] R.S. Ward and R.O. Wells, Twistor Geometry and Field Theory, Cambridge University Press, Cambridge U.K. (1991).

[27] R. Shaw, A new view of $D=7$ Dirac algebra, J. Phys. A 21 (1988) 7 [inSPIRE].

[28] R. Shaw, Finite geometry, Dirac groups and the table of real Clifford algebras, in Clifford Algebras and Spinor Structures, R. Ablamowicz and P. Lounesto eds., Kluwer Academic Publishers, Dordrecht Netherlands, pg. 59.

[29] W. Dür, G. Vidal and J. Cirac, Three qubits can be entangled in two inequivalent ways, Phys. Rev. A 62 (2000) 062314 [inSPIRE].

[30] M. Duff and S. Ferrara, $E_{7}$ and the tripartite entanglement of seven qubits, Phys. Rev. D 76 (2007) 025018 [quant-ph/0609227] [INSPIRE].

[31] P. Vrana and P. Lévay, The Veldkamp space of multiple qubits, J. Phys. A 43 (2010) 125303 [arXiv:0906.3655] [INSPIRE].

[32] H. Pralle, The hyperplanes of DW(5,2), Experiment. Math. 14 (2005) 373.

[33] J.L. Clerc, Special prehomogeneous vector spaces associated to $F_{4}, E_{6}, E_{7}, E_{8}$ and simple Jordan algebras of rank 3, J. Algebra 264 (2003) 98.

[34] I.M. Gel'fand, M.M. Kapranov and A.V. Zelevinsky, Discriminants, Resultants and Multidimensional Determinants, Birkhäuser, Boston U.S.A. (1994).

[35] W.L. Edge, An orthogonal group of order $2^{13} \cdot 3^{5} .5^{2} .7$, Ann. Mat. Pura Appl. 61 (1963) 1.

[36] M. Saniga, P. Lévay and P. Pracna, Charting the Real Four-Qubit Pauli Group via Ovoids of a Hyperbolic Quadric of PG(7,2), J. Phys. A 45 (2012) 295304 [arXiv:1202.2973] [INSPIRE].

[37] A.E. Schroth, How to draw a hexagon, Discr. Math. 199 (1999) 161.

[38] B. Polster, A.E. Schroth and H. van Maldeghem, Generalized flatland, Math. Intell. 23 (2001) 33.

[39] H. van Maldeghem, Monographs in Mathematics. Vol. 93: Generalized Polygons, Birkhäuser, Basel Switzerland (1998).

[40] B.N. Cooperstein, Maximal subgroups of $G_{2}\left(2^{n}\right)$, J. Algebra 70 (1981) 23.

[41] L. Manivel, Configurations of lines and models of Lie algebras, J. Algebra 304 (2006) 457. 
[42] A. Elduque, The Magic Square and Symmetric Compositions II, Rev. Mat. Iberoamericana 23 (2007) 57.

[43] B.N. Cooperstein, A note on the Weyl group of type E7, Europ. J. Comb. 11 (1990) 415.

[44] B.N. Cooperstein, The Fifty-Six-Dimensional Module for $E_{7}$ : I. A Four Form for $E_{7}, J$. Algebra 173 (1995) 361.

[45] P. Breitenlohner, D. Maison and G.W. Gibbons, Four-Dimensional Black Holes from Kaluza-Klein Theories, Commun. Math. Phys. 120 (1988) 295 [InSPIRE].

[46] P. Breitenlohner and D. Maison, On nonlinear $\sigma$-models arising in (super-)gravity, Commun. Math. Phys. 209 (2000) 785 [gr-qc/9806002] [inSPIRE].

[47] G. Bossard, H. Nicolai and K. Stelle, Universal BPS structure of stationary supergravity solutions, JHEP 07 (2009) 003 [arXiv: 0902.4438] [INSPIRE].

[48] G. Bossard and H. Nicolai, Multi-black holes from nilpotent Lie algebra orbits, Gen. Rel. Grav. 42 (2010) 509 [arXiv:0906.1987] [INSPIRE].

[49] G. Bossard, Y. Michel and B. Pioline, Extremal black holes, nilpotent orbits and the true fake superpotential, JHEP 01 (2010) 038 [arXiv: 0908.1742] [INSPIRE].

[50] D.A. Richter, Gossets figure in a Clifford algebra, Adv. Appl. Clifford Algebras 14 (2004) 214.

[51] P. Lévay, STU Black Holes as Four Qubit Systems, Phys. Rev. D 82 (2010) 026003 [arXiv: 1004.3639] [INSPIRE].

[52] J. Ehlers, Konstruktionen und Charakterisierung von Lösungen der Einsteinschen Gravitationsfeldgleichungen, Ph.D. Thesis, Hamburg University, Hamburg Germany (1957).

[53] R.P. Geroch, A Method for generating solutions of Einstein's equations, J. Math. Phys. 12 (1971) 918 [INSPIRE]. 